Part of Journal of Research of the National Bureau of Standards, Volume 21, August 1938

\title{
EXTENSION AND REVISION OF THE ARC SPECTRUM OF SILICON
}

\author{
By Carl C. Kiess
}

\section{ABSTRACT}

A new survey has been made of the spectrum emitted by neutral silicon atoms. Revised wave lengths and intensities are given for 400 lines between $1565 \mathrm{~A}$ in the ultraviolet and $12270 \mathrm{~A}$ in the infrared. The fine structure of some red and infrared lines that appear diffuse when emitted by the arcs in air has been resolved by enclosing the light source in an atmosphere of nitrogen at reduced pressure. Nearly all the lines have been classified as combinations between known and new singlet and triplet terms required by atomic theory. These terms belong to various series of three or more members each and yield accordant results for the value of the ground state $3 p^{3} \mathrm{P}$ 。 of $\mathrm{Si}$. . This value, $65743 \mathrm{~cm}^{-1}$, corresponds to an ionization potential of 8.11 volts. Most of the observed silicon lines longer than $3000 \mathrm{~A}$ and many that are not emitted in the arc but are predictable from the terms, correspond to faint Fraunhofer lines heretofore unidentified.

\section{CONTENTS}

I. Introduction

185

II. Experimental procedure

III. Results

1. Wave lengths and intensities 187

2. Term structure of Si I__.

3. Series and ionization potential of Si I

4. Silicon in the sun

5. Lines of Si II

\section{INTRODUCTION}

The published descriptions of the arc spectrum of silicon show that its principal features appear in the ultraviolet and in the infrared. Only a few lines lie in the visible region. Fowler's ${ }^{1}$ work, nearly 10 years ago, brought to light an extensive spectrum, in the Schumann region, that results from combinations between the ground states of the atom and various higher energy states. Several years later this work was supplemented, at the National Bureau of Standards, ${ }^{2}$ by an extension of the spectrum into the infrared as far as the photographic methods would allow. Since that time new types of photographic plates, brought out by the Eastman Kodak Research Laboratories, ${ }^{3}$ have made it possible to record the spectrum out to $12270 \mathrm{~A}$, nearly $1000 \mathrm{~A}$ beyond the limit reached in the earlier investigation. The new data have led to new classifications of the arc lines of silicon and have suggested corrections of older classifications. As a consequence of the new findings, a resurvey of the entire spectrum has been made, the results of which are presented below.

1 A. Fowler, Proc. Roy. Soc. (London) [A] 123, 422 (1929).
2 C. C. Kiess, BS J. Research 11, 775 (1933) RP624.

${ }^{3}$ L. S. G. Brooker and G. H. Keyes, J. Franklin Inst. 219, 255 (1935). 


\section{EXPERIMENTAL PROCEDURE}

The prism and grating spectrographs of the National Bureau of Standards were employed to photograph the arc spectrum of silicon from wave length $1976 \mathrm{~A}$, in the ultraviolet, to $12270 \mathrm{~A}$ in the infrared. The region from 2124 to 1976 A was recorded with the Hilger E1 spectrograph, which gives a dispersion ranging from $1.5 \mathrm{~A} / \mathrm{mm}$ to $1 \mathrm{~A} / \mathrm{mm}$ in this region. The more powerful prism spectrograph, also by Hilger, and carrying a large $60^{\circ}$ Cornu prism in combination with a $30^{\circ}$ reflecting prism, in the $\mathrm{E} 185$ mounting, was used in the range from 3000 to $2120 \mathrm{~A}$, the dispersion varying from $2 \mathrm{~A} / \mathrm{mm}$ to $0.4 \mathrm{~A} / \mathrm{mm}$. The gratings used for the photography of the longer waves were the ones ruled by Anderson with 7,500 lines per inch and the one by Rowland with 20,000 lines per inch. Adequate descriptions of them have been given elsewhere. ${ }^{4}$ When the observational work described in this paper was nearing completion, a third grating, by R. W. Wood, ${ }^{5}$ ruled with 30,000 lines per inch on an aluminized Pyrex mirror, became available, and was used to photograph the spectrum from 2200 to 8600 A.

The light source with which most of the observations were made was the arc in air between lumps of commercial silicon about 95 percent pure. The currents in the arc ranged from 6 to 15 amperes supplied from a 220-volt d-c circuit. Many of the silicon arc lines in the red and infrared are diffuse and hazy when emitted by the arc in air and are always superimposed upon a background made up of a mixture of impurity lines and band lines due to SiO. These conditions make it impossible to detect the close and faint satellites that accompany some of the stronger lines. Accordingly, a series of observations was made with the silicon arc enclosed in atmospheres of nitrogen at various low pressures ranging from 25 to $350 \mathrm{~mm}$ of $\mathrm{Hg}$. The arc chamber was a reproduction of that described and illustrated by Curtis. $^{6}$ In order to keep the low-pressure observations free from blends with lines of impurities a search for highly purified silicon was instigated, and when the problem was brought to the attention of R. L. Templin, he very generously donated for the work a piece of the cast silicon rod described by him. ${ }^{7}$ Later, when more electrode material was needed some highly purified silicon granules were made available by J. H. Critchett, of the Union Carbide Research Laboratories. This material was alloyed with a small amount of pure silver and cast into rods through the courtesy of W. H. Swanger and A. J. Dornblatt of the Metallurgical Division of this Bureau.

In addition to the observations described above, there were available for measurement several spectrograms covering the Schumann region. These observations were made especially for this investigation by A. G. Shenstone of Princeton University, with the normal-incidence vacuum spectrograph of the Palmer Physical Laboratory. This instrument carries a 2-m glass grating ruled with 30,000 lines to the inch by Wood, and gives a dispersion of $4.2 \mathrm{~A} / \mathrm{mm}$. The source was an arc of approximately 3 amperes between electrodes of metallic silicon cut from the piece supplied by Templin and operated in pure nitrogen at atmospheric pressure. No trace of the confusing silicon

4 W. F. Meggers and K. Burns, BS Sci. Pap. 18, 191 (1922) S441.

5 R. W. Wood, Nature 140, 723 (1937).

6 H. D. Curtis, J. Opt. Soc. Am. 8, 697 (1924)

7 R. L. Templin, Metals and Alloys 3, 136 (1932). 
oxide bands appears on any of the exposures, which ranged from 2 to 75 minutes' duration.

Measurement of all the plates described above was done with the comparators at the National Bureau of Standards. All the spectrograms made at the Bureau bore also the iron or copper spectra to furnish the reference standards for the reduction of the wave lengths; but, in general, there were sufficient numbers of iron lines present as impurities in the silicon exposures to serve as internal standards of reference. The wave lengths of the reference lines were those that have been adopted as international secondary standards ${ }^{8}$ or were selected from the lists of Burns and Walters. ${ }^{\circ}$

For the Princeton plates no comparison spectrum was available. The preliminary wave lengths, derived from the nitrogen and carbon lines that were present as impurities among the silicon lines, were subsequently corrected by amounts derived from term values based on the measurements of National Bureau of Standards spectrograms of silicon.

\section{RESULTS}

\section{WAVE LENGTHS AND INTENSITIES}

Only those wave lengths of silicon that have actually been measured at the National Bureau of Standards are given in the first column of tables 1 and 2. For most of the lines these values represent the means of 2 to 10 determinations. For the diffuse and hazy lines no great precision is claimed and errors as great as $0.10 \mathrm{~A}$ may be present. For the sharper lines, however, that were measured on the highdispersion spectrograms, it is believed that the errors are not greater than $0.01 \mathrm{~A}$. This is indicated by a comparison of the strong ultraviolet lines with the interferometer observations made at the Allegheny Observatory, ${ }^{10}$ which shows a mean difference of $+0.003 \mathrm{~A}$ between the two sets of determinations.

TABLE 1.-Wave lengths in the arc spectrum of silicon emitted by the arc-in-air

\begin{tabular}{|c|c|c|c|c|}
\hline$\lambda_{\mathrm{air}} \mathrm{A}$ & Intensity & $\lambda_{\text {sun }}$ & $\nu_{\mathrm{vac}} \mathrm{cm}^{-1}$ & Term combination \\
\hline $\begin{array}{l}12270.50 \\
12103.46 \\
12031.49 \\
11991.57 \\
11984.20\end{array}$ & $\begin{array}{r}2 \\
5 \\
25 \\
10 \\
20\end{array}$ & 3. 47 & $\begin{array}{l}8147.40 \\
8259.84 \\
8309.25 \\
8336.91 \\
8342.04\end{array}$ & $\begin{array}{l}4 s^{3} \mathrm{P}_{2}^{\circ}-4 p p^{3} \mathrm{D}_{2} \\
4 s^{3} \mathrm{P}_{1}^{\circ}-4 p p^{3} \mathrm{D}_{1} \\
4 s^{3} \mathrm{P}_{2}^{\circ}-4 p p^{3} \mathrm{D}_{3} \\
4 s^{3} \mathrm{P}_{0}^{\circ}-4 p p^{3} \mathrm{D}_{1} \\
4 s^{3} \mathrm{P}_{1}^{\circ}-4 p^{3} \mathrm{D}_{2}\end{array}$ \\
\hline $\begin{array}{l}11821.80 \\
11640.58 \\
11611.49 \\
11607.42\end{array}$ & $\begin{array}{l}0 b \\
2 b \\
5 b \\
0 b\end{array}$ & 1. 65 & $\begin{array}{l}8456.64 \\
8588.31 \\
8609.80 \\
8612.82\end{array}$ & $\begin{array}{l}3 d^{3} \mathrm{P}_{0}^{\circ}-4 f^{3} \mathrm{D}_{1} \\
3 d^{3} \mathrm{P}_{2}^{\circ}-4 f^{3} \mathrm{D}_{3} \\
4 p^{1} \mathrm{D}_{2}-4 d^{1} \mathrm{P}_{1}\end{array}$ \\
\hline 11591.98 & $4 b$ & 2. 27 & 8624.30 & $\begin{array}{l}3 d^{3} \mathrm{P}_{1}^{0}-4 f^{3} \mathrm{D}_{2} \\
3 d^{3} \mathrm{P}_{1}^{\circ}-4 f^{3} \mathrm{D}_{1}\end{array}$ \\
\hline $\begin{array}{l}11502.94 \\
11485.68 \\
11468.54 \\
11308.45 \\
11290.01\end{array}$ & $\begin{array}{r}3 b \\
2 b \\
1 b \\
2 b \\
10 b\end{array}$ & & $\begin{array}{l}8691.05 \\
8704.11 \\
8717.12 \\
8840.52 \\
8854.96\end{array}$ & $\begin{array}{l}3 d^{3} \mathrm{P}_{2}^{\circ}-4 f^{3} \mathrm{D}_{2} \\
4 p^{1} \mathrm{D}_{2}-4 d^{1} \mathrm{~F}_{3} \\
3 d^{3} \mathrm{~F}_{4}^{\circ}-4 f^{3} \mathrm{~F}_{4} \\
3 d^{3} \mathrm{~F}_{3}^{\circ}-4 f^{3} \mathrm{~F}_{2} \\
3 d^{3} \mathrm{~F}_{3}^{\circ}-4 f^{3} \mathrm{~F}_{4}\end{array}$ \\
\hline
\end{tabular}

8 Trans. Int. Astron. Union 3, 77 (1928).

$9 \mathrm{~K}$. Burns and F. M. Walters, Pub. Allegheny[Obs. 8, 27 (1930); 8, 39 (1931).

10 N. E. Wagman, Univ. Pittsburgh Bul. 34, 333.(1937). F. J. Sullivan, Univ. Pittsburgh Bul. (in press). $79859-38-5$ 
TABLE 1.-Wave lengths in the arc spectrum of silicon emitted by the arc-in-air-Con.

\begin{tabular}{|c|c|c|c|c|}
\hline$\lambda_{\mathrm{air}} \mathbf{A}$ & Intensity & $\lambda_{\text {sun }}$ & $\nu_{\mathrm{vac}} \mathrm{cm}^{-1}$ & Term combination \\
\hline $\begin{array}{l}11254.97 \\
11253.22 \\
11202.02 \\
11187.74 \\
11130.37\end{array}$ & $\begin{array}{r}3 b \\
2 b \\
1 b \\
20 b \\
7 b\end{array}$ & & $\begin{array}{l}8882.53 \\
8883.91 \\
8924.52 \\
8935.91 \\
8981.97\end{array}$ & $\begin{array}{l}3 d^{3} \mathrm{~F}_{2}^{\circ}-4 f^{3} \mathrm{~F}_{2} \\
3 d^{3} \mathrm{~F}_{2}^{\circ}-4 f^{3} \mathrm{~F}_{3} \\
3 d^{3} \mathrm{~F}_{4}^{\circ}-4 f^{3} \mathrm{G}_{5}^{\circ}\end{array}$ \\
\hline $\begin{array}{l}11018.00 \\
11013.31 \\
10984.24 \\
10982.28 \\
10979.27\end{array}$ & $\begin{array}{l}70 \\
1 b \\
3 b \\
7 b \\
35\end{array}$ & $\begin{array}{l}8.23 \\
4.42 \\
2.39 \\
9.34\end{array}$ & $\begin{array}{l}9073.58 \\
9077.44 \\
9101.47 \\
9103.09 \\
9105.59\end{array}$ & $\begin{array}{r}3 d^{3} \mathrm{~F}_{3}^{\circ}-4 f^{3} \mathrm{G}_{3} \\
3 d^{3} \mathrm{~F}_{3}-4 f^{3} \mathrm{G}_{4} \\
4 s^{3} \mathrm{P}_{2}^{\circ}-4 p^{3} \mathrm{P}_{1}\end{array}$ \\
\hline $\begin{array}{l}10889.15 \\
10885.16 \\
10882.66 \\
10869.54 \\
10844.02\end{array}$ & $\begin{array}{c}0 b \\
10 b \\
5 b \\
125 \\
25 b\end{array}$ & $\begin{array}{l}5.37 \\
2.84 \\
9.57 \\
3.89\end{array}$ & $\begin{array}{l}9180.94 \\
9184.30 \\
9186.41 \\
9197.50 \\
9219.15\end{array}$ & $\begin{array}{c}3 d^{3} \mathrm{~F}_{2}^{\circ}-4 f^{3} \mathrm{G}_{3} \\
4 p^{3} \mathrm{D}_{3}-4 d^{3} \mathrm{~F}_{3}^{\circ} \\
4 s^{1} \mathrm{P}_{1}^{\circ}-4 p^{1} \mathrm{D}_{2} \\
4 p^{1} \mathrm{P}_{1}-4 d^{1} \mathrm{D}_{2}^{\circ}\end{array}$ \\
\hline $\begin{array}{l}\text { 10827. } 09 \\
10807.75 \\
10796.52 \\
10786.86 \\
10784.33\end{array}$ & $\begin{array}{c}100 \\
1 h \\
0 h \\
50 \\
5 b\end{array}$ & $\begin{array}{l}\text { 7. } 14 \\
\\
6.85 \\
4.58\end{array}$ & $\begin{array}{l}9233.57 \\
9250.09 \\
9259.70 \\
9268.00 \\
9270.18\end{array}$ & $\begin{array}{l}4 s^{3} \mathrm{P}_{2}^{\circ}-4 p^{3} \mathrm{P}_{2} \\
3 d^{3} \mathrm{~F}_{2}^{\circ}-4 f^{1} \mathrm{D}_{2} \\
4 s^{3} \mathrm{P}_{1}^{\circ}-4 p^{3} \mathrm{P}_{0} \\
4 p^{3} \mathrm{D}_{2}-4 d^{3} \mathrm{~F}_{2}^{\circ}\end{array}$ \\
\hline $\begin{array}{l}\text { 10768. } 35 \\
10749.40 \\
10727.21 \\
10694.14 \\
10689.52\end{array}$ & $\begin{array}{l}0 h \\
60 \\
75 b \\
50 b \\
20 b\end{array}$ & $\begin{array}{l}\text { 9. } 37 \\
\text { 7. } 41 \\
\text { 4. } 23 \\
9.73\end{array}$ & $\begin{array}{l}9283.93 \\
9300.30 \\
9319.53 \\
9348.35 \\
9352.39\end{array}$ & $\begin{array}{l}4 s^{3} \mathrm{P}_{1}-4 p^{3} \mathrm{P}_{1} \\
4 p^{3} \mathrm{D}_{3}-4 d^{3} \mathrm{~F}_{4}^{\circ} \\
4 p^{3} \mathrm{D}_{2}-4 d^{3} \mathrm{~F}_{3} \\
4 p^{3} \mathrm{D}_{1}-4 d^{3} \mathrm{~F}_{2}^{\circ}\end{array}$ \\
\hline $\begin{array}{l}10660.98 \\
10627.81 \\
10603.38 \\
10585.12 \\
10582.66\end{array}$ & $\begin{array}{c}50 \\
20 b \\
60 \\
100 \\
1\end{array}$ & $\begin{array}{l}0.98 \\
7.63 \\
\text { 3. } 44 \\
\text { 5. } 165\end{array}$ & $\begin{array}{l}9377.43 \\
9406.70 \\
9428.38 \\
9444.64 \\
9446.83\end{array}$ & $\begin{array}{l}4 s^{3} \mathrm{P}_{0}^{\circ}-4 p^{3} \mathrm{P}_{1} \\
4 p^{1} \mathrm{P}_{1}-4 d^{3} \mathrm{P}_{2}^{\circ} \\
4 s^{3} \mathrm{P}_{1}^{\circ}-4 p^{3} \mathrm{P}_{2} \\
4 s^{3} \mathrm{P}_{2}^{\circ}-4 p^{3} \mathrm{~S}_{1} \\
4 p^{1} \mathrm{D}_{2}-6 s^{1} \mathrm{P}_{1}^{\circ}\end{array}$ \\
\hline $\begin{array}{l}10498.86 \\
10371.23 \\
10288.83 \\
10155.88 \\
10100.84\end{array}$ & $\begin{array}{r}2 \\
50 \\
25 \\
1 \\
1\end{array}$ & $\begin{array}{l}\text { 1. } 285 \\
8.950 \\
6.16\end{array}$ & $\begin{array}{l}9522.24 \\
9639.42 \\
9716.62 \\
9843.81 \\
9897.45\end{array}$ & $\begin{array}{l}4 s^{3} \mathrm{P}_{1}^{\circ}-4 p{ }^{3} \mathrm{~S}_{1} \\
4 s^{3} \mathrm{P}_{0}^{\circ}-4 p p^{3} \mathrm{~S}_{1} \\
4 p^{3} \mathrm{P}_{2}-5 d^{3} \mathrm{D}_{2}^{\circ}\end{array}$ \\
\hline $\begin{array}{r}10067.84 \\
10020.16 \\
10015.33 \\
10009.82 \\
9967.46\end{array}$ & $\begin{array}{l}4 b \\
1 \\
1 \\
1 \\
1\end{array}$ & & $\begin{array}{r}9929.90 \\
9977.15 \\
9981.96 \\
9987.45 \\
10029.90\end{array}$ & $\begin{array}{c}4 p^{3} \mathrm{P}_{2}-5 d^{3} \mathrm{D}_{3}^{\circ} \\
3 d^{1} \mathrm{D}_{2}^{\circ}-5 p^{3} \mathrm{P}_{1} \\
4 p{ }^{1} \mathrm{~S}_{0}-5 d^{1} \mathrm{P}_{1}^{\circ} \\
4 p^{3} \mathrm{P}_{0}-5 d^{3} \mathrm{D}_{1}^{\circ}\end{array}$ \\
\hline $\begin{array}{l}9913.16 \\
9891.90 \\
9886.92 \\
9839.58 \\
9789.24\end{array}$ & $\begin{array}{l}1 b \\
5 b \\
2 b \\
2 b \\
2 h\end{array}$ & $\begin{array}{l}\text { 1. } 61 \\
\text { 7. } 06 \\
9.36\end{array}$ & $\begin{array}{l}10084.84 \\
10106.51 \\
10111.60 \\
10160.25 \\
10212.50\end{array}$ & $\begin{array}{c}4 p^{3} \mathrm{P}_{2}-6 s^{3} \mathrm{P}_{1}^{\circ} \\
4 p{ }^{3} \mathrm{~S}_{1}-6 s^{3} \mathrm{P}_{2}^{\circ} \\
4 p{ }^{1} \mathrm{D}_{2}-5 d^{1} \mathrm{D}_{2}^{\circ} \\
4 p{ }^{3} \mathrm{P}_{1}-6 s^{3} \mathrm{P}_{0}^{\circ} \\
4 p^{3} \mathrm{P}_{1}-6 s^{3} \mathrm{P}_{1}^{\circ}\end{array}$ \\
\hline $\begin{array}{l}9770.10 \\
9768.27 \\
9758.08 \\
9738.60 \\
9689.41\end{array}$ & $\begin{array}{l}4 b \\
5 b \\
2 h \\
6 b \\
8 b\end{array}$ & 0.30 & $\begin{array}{l}\text { 10232. } 51 \\
10234.42 \\
10245.11 \\
10265.61 \\
10317.72\end{array}$ & $\begin{array}{l}4 s^{3} \mathrm{P}_{2}^{\circ}-4 p{ }^{1} \mathrm{D}_{2} \\
4 p^{3} \mathrm{P}_{0}-6 s^{3} \mathrm{P}_{1}^{\circ} \\
4 p^{3} \mathrm{P}_{2}-6 s^{3} \mathrm{P}_{2}^{\circ}\end{array}$ \\
\hline
\end{tabular}


TABLE 1.-Wave lengths in the arc spectrum of silicon emitted by the arc-in-air-Con.

\begin{tabular}{|c|c|c|c|c|}
\hline$\lambda_{\mathrm{air}} \mathrm{A}$ & Intensity & $\lambda_{\text {sun }}$ & $\nu_{\mathrm{vac}} \mathrm{cm}^{-1}$ & Term combination \\
\hline 9585.72 & 4 & 5. 70 & 10429. 33 & $4 s^{3} \mathrm{P}_{1}^{\circ}-4 p^{1} \mathrm{D}_{2}$ \\
\hline 9570.08 & 4 & 9. 93 & 10446. 37 & $\begin{array}{l}3 d^{1}{ }^{1} \mathrm{D}_{2}^{\circ}-5 p^{1} \mathrm{D}_{2} \\
4 n^{3} \mathrm{P}_{1}-6{ }^{3} \mathrm{P}_{0}\end{array}$ \\
\hline 9505. 28 & 5 & & 10517. 60 & $4 p^{3} \mathrm{~S}_{1}-5 d^{3} \mathrm{P}_{2}^{0}$ \\
\hline 9421.82 & 4 & & 10610. 75 & $4 p^{3} \mathrm{~S}_{1}-5 d^{3} \mathrm{P}_{1}$ \\
\hline 9413. 59 & 200 & 3.51 & 10620.03 & $4 s^{1} \mathrm{P}_{\mathrm{i}}^{\circ}-4 p{ }^{1} \mathrm{~S}_{0}$ \\
\hline 9393. 40 & $2 b$ & & 10642. 86 & $4 p^{3} \mathrm{~S}_{1}-5 d^{3} \mathrm{P}_{0}^{0}$ \\
\hline $\begin{array}{l}9318.24 \\
9254.59\end{array}$ & $\begin{array}{l}4 \\
4 h\end{array}$ & 8.18 & $\begin{array}{l}10728.70 \\
10802.49\end{array}$ & $4 p^{3} \mathrm{P}_{2}-5 d^{3} \mathrm{P}_{2}^{0}$ \\
\hline 9238.60 & $2 h$ & & 10821. 18 & $4 p^{3} \mathrm{P}_{2}-5 d^{3} \mathrm{P}_{1}^{\circ}$ \\
\hline 9209.66 & $1 b$ & & 10855. 19 & \\
\hline 9208. 55 & $5 b$ & 8. 60 & 10856.50 & $4 p^{3} \mathrm{P}_{1}-5 d^{3} \mathrm{P}_{2}^{\circ}$ \\
\hline 9147.39 & $0 \mathrm{~h}$ & & 10929.08 & $4 p^{3} \mathrm{P}_{1}-5 d^{3} \mathrm{P}_{0}^{0}$ \\
\hline 9103.37 & $3 b$ & & 10981.93 & $4 p^{3} \mathrm{P}_{0}-5 d^{3} \mathrm{P}_{1}^{0}$ \\
\hline $\begin{array}{l}9064.06 \\
9052.57\end{array}$ & $\begin{array}{l}0 b l \\
0 b l\end{array}$ & & $\begin{array}{l}11029.56 \\
11043.56\end{array}$ & $3 d^{3} \mathrm{P}_{1}^{\circ}-5 f^{3} \mathrm{D}_{2}$ \\
\hline 9022.40 & $2 h$ & & 11080.49 & \\
\hline 9009. 04 & $5 h l$ & & 11096. 92 & $\begin{array}{l}3 d^{3} \mathrm{P}_{2}^{\circ}-5 f^{3} \mathrm{D}_{3} \\
3 d^{3} \mathrm{P}_{0}^{0}-5 f^{3} \mathrm{D}_{3}\end{array}$ \\
\hline $\begin{array}{l}\text { 8949. } 33 \\
8925.55 \\
8899.50\end{array}$ & $\begin{array}{r}15 b \\
8 b \\
3 b\end{array}$ & 9. 06 & $\begin{array}{l}11170.96 \\
11200.72 \\
11233.51\end{array}$ & $\begin{array}{l}4 p^{3} \mathrm{D}_{2}-6 s^{3} \mathrm{P}_{1}^{\circ} \\
4 p^{3} \mathrm{D}_{1}-6 s^{3} \mathrm{P}_{0}^{\circ} \\
3 d^{3} \mathrm{~F}_{4}^{\circ}-5 f^{3} \mathrm{~F}_{3}^{\circ}\end{array}$ \\
\hline 8898. 97 & $3 b$ & 8. 99 & 11234.18 & $3 d^{3} \mathrm{~F}_{4}^{\circ}-5 f^{3} \mathrm{~F}_{4}^{\circ}$ \\
\hline 8892. 97 & $25 b$ & 2. 73 & 11241.75 & $4 p^{3} \mathrm{D}_{3}-6 s^{3} \mathrm{P}_{2}^{0}$ \\
\hline 8883.84 & $4 b$ & 3. 68 & 11253. 30 & $4 p^{3} \mathrm{D}_{1}-6 s^{3} \mathrm{P}_{1}^{\circ}$ \\
\hline 8791. 28 & $5 b$ & & 11371. 79 & $\begin{array}{l}3 d^{3} \mathrm{~F}_{3}^{\circ}-5 f^{3} \mathrm{~F}_{2} \\
4 p^{1} \mathrm{D}_{2}-5 d^{1} \mathrm{~F}_{3}\end{array}$ \\
\hline 8790.88 & $4 b$ & & 11372. 31 & $\begin{array}{l}3 d^{3} \mathbf{F}_{3}^{0}-5 f^{3} \mathbf{F}_{4} \\
3 d^{3} \mathbf{F}_{3}^{0}-5 f^{3} \mathbf{F}_{3}\end{array}$ \\
\hline 8766.68 & $3 b$ & 6. 42 & 11403. 70 & $4 p^{3} \mathrm{D}_{2}-6 s^{3} \mathrm{P}_{2}^{\circ}$ \\
\hline 8752.17 & 200 & 2. 02 & 11422. 60 & $3 d^{1} \mathrm{D}_{2}^{\circ}-4 f^{1} \mathrm{~F}_{3}$ \\
\hline 8742.60 & 100 & 2. 47 & 11435. 11 & $3 d^{1} \mathrm{D}_{2}^{\circ}-4 f^{3} \mathrm{~F}_{3}$ \\
\hline 8729.02 & $5 b$ & 9.17 & 11452. 90 & $3 d^{3} \mathrm{~F}_{2}^{0}-5 f^{3} \mathrm{~F}_{2}$ \\
\hline 8728.38 & $10 b$ & 8. 02 & 11453. 74 & $3 d^{3} \mathrm{~F}_{2}-5 f^{3} \mathrm{~F}_{3}$ \\
\hline 8648.89 & $100 h l$ & 8.47 & 11559.00 & \\
\hline 8606. 43 & $1 b$ & 6. 38 & 11616. 03 & $4 p^{3} \mathrm{D}_{1}-6 s^{1} \mathrm{P}_{1}^{\circ}$ \\
\hline 8597. 00 & $2 h l$ & 7. 059 & 11628. 77 & $3 d^{3} \mathrm{~F}_{3}^{\circ}-5 f^{3} \mathrm{G}_{3}$ \\
\hline 8579.15 & $2 b$ & 9.08 & 11652.97 & \\
\hline 8556. 64 & $100 b$ & 6.795 & 11683. 63 & $3 d^{1} \mathrm{D}_{2}^{\circ}-4 f^{3} \mathrm{G}_{3}$ \\
\hline 8536.80 & $2 b$ & 6. 68 & 11710.78 & \\
\hline 8536. 38 & $3 b$ & 6. 16 & 11711.36 & $3 d^{3} \mathrm{~F}_{2}^{\circ}-5 f^{3} \mathrm{G}_{3}$ \\
\hline 8503. 17 & 5 & 3. 145 & 11757. 09 & \\
\hline 8502. 38 & $30 b$ & 2. 228 & 11758.32 & $3 d^{1} \mathrm{D}_{2}^{\circ}-4 f^{3} \mathrm{D}_{3}$ \\
\hline 8501.50 & $20 b$ & 1. 553 & 11759.40 & $3 d^{1} \mathrm{D}_{2}^{\circ}-4 f^{1} \mathrm{D}_{2}$ \\
\hline 8444.48 & $3 b$ & 4. 377 & 11838.81 & $3 d^{1} \mathrm{D}_{2}^{\circ}-4 f^{3} \mathrm{D}_{1}$ \\
\hline 8444.00 & $15 b$ & 3. 975 & 11839. 48 & $3 d^{1} \mathrm{D}_{2}^{0}-4 f^{3} \mathrm{D}_{2}$ \\
\hline 8338.43 & $5 b$ & 8. 343 & 11989. 37 & $4 p^{1} \mathrm{P}_{1}-6 s^{3} \mathrm{P}_{1}^{0}$ \\
\hline 8317.45 & $2 b$ & 7. 394 & 12019. 62 & $3 d^{3} \mathrm{D}_{1}^{0}-5 p^{3} \mathrm{P}_{0}$ \\
\hline 8306.80 & $4 b$ & 6. 699 & 12035.02 & $3 d^{3} \mathrm{D}_{2}^{0}-5 p^{3} \mathrm{P}_{1}$ \\
\hline
\end{tabular}


TABLE 1.-Wave lengths in the arc spectrum of silicon emitted by the arc-in-air-Con.

\begin{tabular}{|c|c|c|c|c|}
\hline$\lambda_{\mathrm{air}} \mathrm{A}$ & Intensity & $\lambda_{\text {sun }}$ & $\nu_{\mathrm{Vac}} \mathrm{cm}^{-1}$ & Term combination \\
\hline $\begin{array}{l}8230.67 \\
8211.48 \\
8093.32 \\
8035.39 \\
7970.91\end{array}$ & $\begin{array}{c}15 \\
2 \\
25 b \\
7 b \\
3 b\end{array}$ & $\begin{array}{l}0.63 \\
\text { 1. } 57 \\
\text { 3. } 232\end{array}$ & $\begin{array}{l}12146.34 \\
12174.73 \\
12352.48 \\
12441.53 \\
12542.17\end{array}$ & $\begin{array}{c}3 d^{3} \mathrm{D}_{3}^{\circ}-5 p{ }^{3} \mathrm{P}_{2} \\
3 d^{3} \mathrm{D}_{2}-5 p^{3} \mathrm{P}_{2} \\
4 p{ }^{1} \mathrm{P}_{1}-6 s^{1} \mathrm{P}_{1} \\
4 p^{3} \mathrm{D}_{3}-5 d^{3} \mathrm{~F}_{3}^{3}\end{array}$ \\
\hline $\begin{array}{l}7970.26 \\
7943.94 \\
7932.20 \\
7918.38 \\
7913.47\end{array}$ & $\begin{array}{r}10 b \\
500 b \\
300 b \\
200 b \\
10 b\end{array}$ & $\begin{array}{l}0.300 \\
\text { 4. } 001 \\
\text { 2. } 351 \\
\text { 8. } 383 \\
\text { 3. } 438\end{array}$ & $\begin{array}{l}12543.20 \\
12584.75 \\
12603.38 \\
12625.38 \\
12633.21\end{array}$ & $\begin{array}{l}4 p^{3} \mathrm{D}_{2}-5 d^{3} \mathrm{~F}_{2} \\
4 p p^{3} \mathrm{D}_{3}-5 d^{3} \mathrm{~F}^{\circ} \\
4 p^{3} \mathrm{D}_{2}-5 d^{3} \mathrm{~F}_{3} \\
4 p^{3} \mathrm{D}_{1}-5 d^{3} \mathrm{~F}_{2} \\
4 p^{1} \mathrm{P}_{1}-5 d^{3} \mathrm{P}_{2}^{\circ}\end{array}$ \\
\hline $\begin{array}{l}7912.55 \\
7850.5 \\
7800.0 \\
7743.2 \\
7742.7\end{array}$ & $\begin{array}{l}3 b \\
2 H \\
4 H \\
4 h \\
5 h\end{array}$ & $\begin{array}{l}2.384 \\
0.88 \\
0.009 \\
2.722\end{array}$ & $\begin{array}{l}12634.68 \\
12734.54 \\
12816.99 \\
12911.01 \\
12911.84\end{array}$ & $\begin{array}{l}4 p^{3} \mathrm{P}_{2}-7 s^{3} \mathrm{P}_{2}^{\circ} \\
3 d^{3} \mathrm{~F}_{3}^{\circ}-6 f^{3} \mathrm{~F}_{2} \\
3 d^{3} \mathrm{~F}_{2}^{\circ}-6 f^{3} \mathrm{~F}_{2}\end{array}$ \\
\hline $\begin{array}{l}7680.35 \\
7424.63 \\
7423.54 \\
7416.00 \\
7415.37\end{array}$ & $\begin{array}{l}100 b \\
20 \\
500 \\
250 \\
15\end{array}$ & $\begin{array}{l}\text { 0. } 267 \\
\text { 4. } 647 \\
\text { 3. } 509 \\
\text { 5. } 958 \\
\text { 5. } 363\end{array}$ & $\begin{array}{l}13016.66 \\
13464.98 \\
13466.96 \\
13480.65 \\
13481.80\end{array}$ & $\begin{array}{l}4 p^{1} \mathrm{P}_{1}-5 d^{1} \mathrm{D}_{2}^{\circ} \\
3 d^{3} \mathrm{D}_{3}^{\circ}-4 f^{3} \mathrm{~F}_{3} \\
3 d^{3} \mathrm{D}_{3}^{\circ}-4 f^{3} \mathrm{~F}_{4} \\
3 d^{3} \mathrm{D}_{2}^{\circ}-4 f^{1} \mathrm{~F}_{3} \\
3 d^{3} \mathrm{D}_{2}^{\circ}-4 f^{3} \mathrm{~F}_{2}\end{array}$ \\
\hline $\begin{array}{l}7409.11 \\
7405.85 \\
7373.02 \\
7290.21 \\
7289.25\end{array}$ & $\begin{array}{l}100 \\
300 \\
10 b \\
10 \\
250\end{array}$ & $\begin{array}{l}\text { 9. } 100 \\
\text { 5. } 700 \\
\text { 3. } 011 \\
\text { 9. } 199\end{array}$ & $\begin{array}{l}\text { 13493. } 19 \\
13499.13 \\
13559.23 \\
13713.25 \\
13715.06\end{array}$ & $\begin{array}{l}3 d^{3} \mathrm{D}_{2}^{\circ}-4 f^{3} \mathrm{~F}_{3} \\
3 d^{3} \mathrm{D}_{1}^{\circ}-4 f^{3} \mathrm{~F}_{2} \\
4 p^{3} \mathrm{D}_{3}-7 s^{3} \mathrm{P}_{2} \\
3 d^{3} \mathrm{D}_{3}^{\circ}-4 f^{3} \mathrm{G}_{3} \\
3 d^{3} \mathrm{D}_{3}^{\circ}-4 f^{3} \mathrm{G}_{4}\end{array}$ \\
\hline $\begin{array}{l}7275.28 \\
7250.69 \\
7235.86 \\
7235.32 \\
7226.20\end{array}$ & $\begin{array}{l}50 \\
40 \\
10 \\
15 \\
20\end{array}$ & $\begin{array}{l}0.661 \\
\text { 5. } 852 \\
\text { 5. } 368 \\
6.231\end{array}$ & $\begin{array}{l}13741.40 \\
13788.00 \\
13816.26 \\
13817.29 \\
13834.73\end{array}$ & $\begin{array}{l}3 d^{3} \mathrm{D}_{2}^{\circ}-4 f^{3} \mathrm{G}_{3} \\
3 d^{3} \mathrm{D}_{3}^{\circ}-4 f^{3} \mathrm{D}_{3} \\
3 d^{3} \mathrm{D}_{2}^{\circ}-4 f^{3} \mathrm{D}_{3} \\
3 d^{3} \mathrm{D}_{2}^{\circ}-4 f^{1} \mathrm{D}_{2} \\
3 d^{3} \mathrm{D}_{1}^{\circ}-4 f^{1} \mathrm{D}_{2}\end{array}$ \\
\hline $\begin{array}{l}7208.20 \\
7193.89 \\
7193.56 \\
7184.89 \\
7184.54\end{array}$ & $\begin{array}{r}1 \\
5 \\
8 \\
10 \\
1\end{array}$ & 4. 930 & $\begin{array}{l}\text { 13869. } 28 \\
13896.86 \\
13897.50 \\
13914.27 \\
13914.92\end{array}$ & $\begin{array}{l}3 d^{3} \mathrm{D}_{3}^{\circ}-4 f^{3} \mathrm{D}_{2} \\
3 d^{3} \mathrm{D}_{2}^{\circ}-4 f^{3} \mathrm{D}_{1} \\
3 d^{3} \mathrm{D}_{2}^{\circ}-4 f^{3} \mathrm{D}_{2} \\
3 d^{3} \mathrm{D}_{1}-4 f^{3} \mathrm{D}_{1} \\
3 d^{3} \mathrm{D}_{1}-4 f^{3} \mathrm{D}_{2}\end{array}$ \\
\hline $\begin{array}{l}7165.62 \\
7164.75 \\
7084.33 \\
7034.96 \\
7026.61\end{array}$ & $\begin{array}{r}100 b \\
2 b \\
2 b \\
50 b \\
2 b\end{array}$ & $\begin{array}{l}\text { 5. } 568 \\
\text { 4. } 736 \\
\text { 4. } 268 \\
\text { 4. } 920 \\
\text { 6. } 635\end{array}$ & $\begin{array}{l}13951.69 \\
13953.40 \\
14111.78 \\
14210.81 \\
14227.69\end{array}$ & $\begin{array}{l}3 d^{1}{ }^{1} \mathrm{D}_{2}-5 f^{1} \mathrm{D}_{2} \\
3 d^{1} \mathrm{D}_{2}^{\circ}-5 f^{3} \mathrm{~F}_{3},{ }^{2} \\
4 p^{3} \mathrm{D}_{3}-6 d^{3} \mathrm{~F}_{3}^{\circ} \\
3 d^{1} \mathrm{D}_{2}^{\circ}-5 f^{3} \mathrm{G}_{3}\end{array}$ \\
\hline $\begin{array}{l}\text { 7017. } 98 \\
7017.68 \\
7005.84 \\
7003.58 \\
6976.53\end{array}$ & $\begin{array}{r}4 b \\
10 b \\
50 b \\
50 b \\
25 b\end{array}$ & $\begin{array}{l}\text { 7. } 999 \\
\text { 7. } 684 \\
\text { 5. } 909 \\
\text { 3. } 590 \\
6.533\end{array}$ & $\begin{array}{l}14245.19 \\
14245.80 \\
14269.87 \\
14274.48 \\
14329.83\end{array}$ & $\begin{array}{l}3 d^{1} \mathrm{D}_{2}^{\circ}-5 f^{3} \mathrm{D}_{3} \\
3 d^{1} \mathrm{D}_{2}-5 f^{3} \mathrm{D}_{2} \\
4 p^{3} \mathrm{D}_{3}-6 d^{3} \mathrm{~F}_{4}^{\circ} \\
4 p^{3} \mathrm{D}_{2}-6 d^{3} \mathrm{~F}_{3}^{\circ} \\
4 p^{3} \mathrm{D}_{1}-6 d^{3} \mathrm{~F}_{2}^{\circ}\end{array}$ \\
\hline $\begin{array}{l}6848.65 \\
6741.74 \\
6722.67 \\
6721.97 \\
6560.68\end{array}$ & $\begin{array}{l}4 b \\
2 b \\
2 b \\
4 b \\
2 h\end{array}$ & $\begin{array}{l}\text { 8. } 578 \\
\text { 1. } 638 \\
\text { 2. } 718 \\
\text { 1. } 853\end{array}$ & $\begin{array}{l}14597.40 \\
14828.88 \\
14870.94 \\
14872.49 \\
15238.12\end{array}$ & $\begin{array}{l}4 p^{1} \mathrm{P}_{1}-7 s^{1} \mathrm{P}_{1}^{\circ} \\
4 p^{1} \mathrm{P}_{1}-6 d^{1} \mathrm{D}_{2}^{\circ} \\
4 p^{3} \mathrm{D}_{1}-7 d^{3} \mathrm{~F}_{2}^{\circ}\end{array}$ \\
\hline
\end{tabular}


TABLE 1.-Wave lengths in the arc spectrum of silicon emitted by the arc-in-air-Con.

\begin{tabular}{|c|c|c|c|c|}
\hline$\lambda_{\mathrm{air}} \mathrm{A}$ & Intensity & $\lambda_{\text {sun }}$ & $\nu_{\mathrm{V} a \mathrm{c}} \mathrm{cm}^{-1}$ & Term combination \\
\hline $\begin{array}{l}6555.20 \\
6527.49 \\
6415.24 \\
6331.92 \\
6254.96\end{array}$ & $\begin{array}{l}2 h \\
3 h \\
4 b \\
2 b \\
2 h\end{array}$ & 1. 971 & $\begin{array}{l}15250.86 \\
15315.60 \\
15583.58 \\
15788.64 \\
15982.90\end{array}$ & $\begin{array}{l}4 p^{3} \mathrm{D}_{2}-7 d^{3} \mathrm{~F}_{3}^{\circ} \\
4 p^{3} \mathrm{D}_{3}-7 d^{3} \mathrm{~F}_{4}^{\circ} \\
3 d^{3} \mathrm{D}_{3}^{\circ}-5 f^{3} \mathrm{~F}_{3}\end{array}$ \\
\hline $\begin{array}{l}6254.25 \\
6244.56 \\
6243.86 \\
6238.38 \\
6237.62\end{array}$ & $\begin{array}{r}25 h \\
10 h \\
10 h \\
2 h \\
5 h\end{array}$ & $\begin{array}{l}\text { 4. } 179 \\
\text { 4. } 483 \\
\text { 3. } 829 \\
\text { 8. } 396\end{array}$ & $\begin{array}{l}\text { 15984. } 71 \\
16009.52 \\
16011.31 \\
16025.38 \\
16027.33\end{array}$ & $\begin{array}{l}3 d^{3} \mathrm{D}_{3}^{\circ}-5 f^{3} \mathrm{~F}_{4} \\
3 d^{3} \mathrm{D}_{2}^{\circ}-5 f^{1} \mathrm{D}_{2} \\
3 d^{3} \mathrm{D}_{2}^{\circ}-5 f^{3} \mathrm{~F}_{3} \\
{ }^{3} d^{3} \mathrm{D}_{1}^{\circ}-5 f^{1} \mathrm{D}_{2}\end{array}$ \\
\hline $\begin{array}{l}6237.34 \\
6155.73 \\
6155.22 \\
6145.08 \\
6142.53\end{array}$ & $\begin{array}{r}5 h \\
2 h \\
20 h \\
10 h \\
5 h\end{array}$ & $\begin{array}{l}\text { 7. } 334 \\
\text { 5. } 715 \\
\text { 5. } 148 \\
\text { 5. } 030 \\
\text { 2. } 499\end{array}$ & $\begin{array}{l}16028.05 \\
16240.54 \\
16241.89 \\
16268.69 \\
16275.44\end{array}$ & $\begin{array}{l}3 d^{3} \mathrm{D}_{1}-5 f^{3} \mathrm{~F}_{2} \\
3 d^{3} \mathrm{D}_{3}^{\circ}-5 f^{3} \mathrm{G}_{3} \\
3 d^{3} \mathrm{D}_{3}^{\circ}-5 f^{3} \mathrm{G}_{4} \\
3 d^{3} \mathrm{D}_{2}^{\circ}-5 f^{3} \mathrm{G}_{3} \\
3 d^{3} \mathrm{D}_{3}^{\circ}-5 f^{3} \mathrm{D}_{3}\end{array}$ \\
\hline $\begin{array}{l}6131.86 \\
6131.54 \\
6125.03 \\
6124.85 \\
6113.12\end{array}$ & $\begin{array}{l}5 h \\
4 h \\
4 h \\
2 h \\
1 h\end{array}$ & $\begin{array}{l}\text { 1. } 868 \\
\text { 1. } 583 \\
\text { 5. } 032 \\
\text { 4. } 83 \\
\text { 3. } 136\end{array}$ & $\begin{array}{l}16303.76 \\
16304.61 \\
16321.94 \\
16322.42 \\
16353.74\end{array}$ & $\begin{array}{l}3 d^{3} \mathrm{D}_{2}^{\circ}-5 f^{3} \mathrm{D}_{3} \\
3 d^{3} \mathrm{D}_{2}-5 f^{3} \mathrm{D}_{2} \\
3 d^{3} \mathrm{D}_{1}-5 f^{3} \mathrm{D}_{2} \\
3 d^{3} \mathrm{D}_{1}-5 f^{3} \mathrm{D}_{1}\end{array}$ \\
\hline $\begin{array}{l}6106.70 \\
5948.584 \\
5797.912 \\
5793.128 \\
5780.452\end{array}$ & $\begin{array}{c}1 h \\
100 \\
40 \\
30 \\
25\end{array}$ & $\begin{array}{l}\text { 6. } 635 \\
8.552 \\
\text { 7. } 869 \\
3.083 \\
0.390\end{array}$ & $\begin{array}{l}16370.94 \\
16806.08 \\
17242.82 \\
17257.06 \\
17294.90\end{array}$ & $\begin{array}{l}4 s^{1} \mathrm{P}_{1}-5 p{ }^{1} \mathrm{D}_{2} \\
4 s^{3} \mathrm{P}_{2}^{\circ}-5 p^{3} \mathrm{D}_{3} \\
4 s^{3} \mathrm{P}_{1}^{\circ}-5 p p^{3} \mathrm{D}_{2} \\
4 s^{3} \mathrm{P}_{0}^{\circ}-5 p^{3} \mathrm{D}_{1}\end{array}$ \\
\hline $\begin{array}{l}5772.258 \\
5754.258 \\
5708.437 \\
5701.138 \\
5690.470\end{array}$ & $\begin{array}{l}50 \\
8 b \\
75 \\
25 \\
40\end{array}$ & $\begin{array}{l}\text { 2. } 152 \\
\text { 8. } 408 \\
\text { 1. } 111 \\
0.435\end{array}$ & $\begin{array}{l}17319.45 \\
17373.63 \\
17513.08 \\
17535.50 \\
17568.38\end{array}$ & $\begin{array}{l}4 s^{1} \mathrm{P}_{1}^{\circ}-5 p{ }^{1} \mathrm{~S}_{0} \\
4 s^{3} \mathrm{P}_{2}^{0}-5 p p^{3} \mathrm{P}_{1} \\
4 s^{3} \mathrm{P}_{2}^{0}-5 p^{3} \mathrm{P}_{2} \\
4 s^{3} \mathrm{P}_{1}^{\circ}-5 p p^{3} \mathrm{P}_{0} \\
4 s^{3} \mathrm{P}_{1}^{\circ}-5 p^{3} \mathrm{P}_{1}\end{array}$ \\
\hline $\begin{array}{l}5684.523 \\
5665.601 \\
5645.665 \\
5622.23 \\
4102.926\end{array}$ & $\begin{array}{r}50 \\
25 \\
25 \\
3 \\
25\end{array}$ & $\begin{array}{l}\text { 4. } 496 \\
\text { 5. } 566 \\
\text { 5. } 621 \\
\text { 2. } 237 \\
\text { 2. } 945\end{array}$ & $\begin{array}{l}\text { 17586. } 76 \\
17645.49 \\
17707.80 \\
17781.61 \\
24366.00\end{array}$ & $\begin{array}{l}4 s^{3} \mathrm{P}_{2}^{\circ}-5 p^{3} \mathrm{~S}_{1} \\
4 s^{3} \mathrm{P}_{0}^{0}-5 p^{3} \mathrm{P}_{1} \\
4 s^{3} \mathrm{P}_{1}^{\circ}-5 p^{3} \mathrm{P}_{2} \\
4 s^{3} \mathrm{P}_{1}^{\circ}-5 p^{3} \mathrm{~S}_{1} \\
3 p^{1} \mathrm{~S}_{0}-4 s^{3} \mathrm{P}_{1}^{\circ}\end{array}$ \\
\hline $\begin{array}{l}3905.527 \\
2987.65 \\
2970.35 \\
2881.595 \\
2842.35\end{array}$ & $\begin{array}{c}100 \\
25 \\
15 \\
200 r \\
3\end{array}$ & 5. 534 & $\begin{array}{l}25597.52 \\
33461.39 \\
33656.27 \\
34692.86 \\
35171.84\end{array}$ & $\begin{array}{r}3 p{ }^{1} \mathrm{~S}_{0}-4 s^{1} \mathbf{P}_{1} \\
3 p{ }^{1} \mathrm{D}_{2}-4 s^{3} \mathbf{P}_{1}^{\circ} \\
3 p{ }^{1} \mathrm{D}_{2}-4 s^{3} \mathbf{P}_{2}^{\circ} \\
3 p{ }^{1} \mathrm{D}_{2}-4 s^{1} \mathbf{P}_{1}^{\circ} \\
3 p^{1} \mathrm{~S}_{0}-3 d^{3} \mathbf{P}_{1}^{\circ}\end{array}$ \\
\hline $\begin{array}{l}2631.28 \\
2577.13 \\
2568.63 \\
2564.82 \\
2563.67\end{array}$ & $\begin{array}{l}50 r \\
10 \\
15 \\
3 \\
4\end{array}$ & & $\begin{array}{l}37993.00 \\
38791.25 \\
38919.60 \\
38977.41 \\
38994.90\end{array}$ & $\begin{array}{c}3 p{ }^{1} \mathrm{~S}_{0}-3 d^{1} \mathrm{P}_{\mathrm{i}} \\
3 p{ }^{1} \mathrm{~S}_{0}-4 d^{3} \mathrm{D}^{3}{ }^{3} \\
3 p p^{1} \mathrm{~S}_{0}-5 s^{3} \mathrm{P}_{1}^{\circ} \\
3 p^{1} \mathrm{D}_{2}-3 d^{3} \mathrm{D}_{1}^{\circ} \\
3 p^{1} \mathrm{D}_{2}-3 d^{3} \mathrm{D}_{2}^{\circ}\end{array}$ \\
\hline $\begin{array}{l}2532.38 \\
2528.513 \\
2524.112 \\
2519.206 \\
2516.111\end{array}$ & $\begin{array}{l}20 \\
175 r \\
125 r \\
100 r \\
250 r\end{array}$ & & $\begin{array}{l}39476.68 \\
39537.05 \\
39605.98 \\
39683.11 \\
39731.92\end{array}$ & $\begin{array}{l}3 p^{1} \mathrm{~S}_{0}-5 s^{1} \mathrm{P}_{1} \\
3 p^{3} \mathrm{P}_{2}-4 s^{3} \mathrm{P}_{1} \\
3 p^{3} \mathrm{P}_{1}-4 s^{3} \mathrm{P}_{0}^{0} \\
3 p^{3} \mathrm{P}_{1}-4 s^{3} \mathrm{P}_{1}^{\circ} \\
3 p^{3} \mathrm{P}_{2}-4 s^{3} \mathrm{P}_{2}^{0}\end{array}$ \\
\hline
\end{tabular}


TABLE 1.-Wave llengths in the arc spectrum of silicon emitted by the arc-in-air-Con.

\begin{tabular}{|c|c|c|c|c|}
\hline$\lambda_{\mathrm{air}} \mathrm{A}$ & Intensity & $\lambda_{\text {sun }}$ & $\nu_{\mathrm{vac}} \mathrm{cm}^{-1}$ & Term combination \\
\hline $\begin{array}{l}2514.320 \\
2506.896 \\
2452.12 \\
2443.37 \\
2438.77\end{array}$ & $\begin{array}{l}100 r \\
150 r \\
20 \\
20 \\
25\end{array}$ & & $\begin{array}{l}\text { 39760. } 22 \\
39878.02 \\
40768.69 \\
40914.68 \\
40991.84\end{array}$ & $\begin{array}{l}3 p^{3} \mathrm{P}_{0}-4 s^{3} \mathrm{P}_{1}^{\circ} \\
3 p^{3} \mathrm{P}_{1}-4 s^{3} \mathrm{P}_{2}^{\circ} \\
3 p^{3} \mathrm{P}_{2}-4 s^{1} \mathrm{P}_{1}^{\circ} \\
3 p^{3} \mathrm{P}_{1}-4 s^{1} \mathrm{P}_{1}^{\circ} \\
3 p^{3} \mathrm{P}_{0}-4 s^{1} \mathrm{P}_{1}^{\circ}\end{array}$ \\
\hline $\begin{array}{l}2435.160 \\
2303.03 \\
2295.40 \\
2291.03 \\
2289.61\end{array}$ & $\begin{array}{c}100 r \\
20 \\
1 \\
7 \\
10\end{array}$ & & $\begin{array}{l}41052.60 \\
43407.67 \\
43551.94 \\
43635.01 \\
43662.07\end{array}$ & $\begin{array}{c}3 p^{1} \mathrm{D}_{2}-3 d^{1} \mathrm{D}_{2}^{\circ} \\
3 p{ }^{1} \mathrm{~S}_{0}-4 d^{1} \mathrm{P}_{1}^{\circ} \\
3 p^{1} \mathrm{D}_{2}-3 d^{3} \mathrm{~F}_{2}^{\circ} \\
3 p^{1} \mathrm{D}_{2}-3 d^{3} \mathrm{~F}_{3}^{\circ} \\
3 p^{1} \mathrm{~S}_{0}-5 d^{3} \mathrm{D}_{1}^{\circ}\end{array}$ \\
\hline $\begin{array}{l}2278.30 \\
2261.70 \\
2259.58 \\
2218.914 \\
2218.052\end{array}$ & $\begin{array}{c}7 \\
1 \\
7 \\
25 r \\
50 r\end{array}$ & & $\begin{array}{l}43878.79 \\
44200.82 \\
44242.28 \\
45053.02 \\
45070.53\end{array}$ & $\begin{array}{c}3 p{ }^{1} \mathrm{~S}_{0}-6 s^{3} \mathrm{P}_{1}^{\circ} \\
3 p{ }^{1} \mathrm{D}_{2}-3 d^{3} \mathrm{P}_{2}^{0} \\
3 p{ }^{1} \mathrm{~S}_{0}-6 s^{1} \mathrm{P}_{1}^{\circ} \\
3 p{ }^{3} \mathrm{P}_{2}-3 d^{3} \mathrm{D}_{1}^{\circ} \\
3 p^{3} \mathrm{P}_{2}-3 d^{3} \mathrm{D}_{2}^{\circ}\end{array}$ \\
\hline $\begin{array}{l}2216.670 \\
2211.737 \\
2210.880 \\
2207.972 \\
2177.30\end{array}$ & $\begin{array}{r}150 r \\
75 r \\
100 r \\
75 r \\
8 b\end{array}$ & & $\begin{array}{l}45098.63 \\
45199.20 \\
45216.72 \\
45276.26 \\
45914.01\end{array}$ & $\begin{array}{l}3 p^{3} \mathrm{P}_{2}-3 d^{3} \mathrm{D}_{3}^{\circ} \\
3 p^{3} \mathrm{P}_{1}-3 d^{3} \mathrm{D}_{1}^{\circ} \\
3 p{ }^{3} \mathrm{P}_{1}-3 d^{3} \mathrm{D}_{2}^{\circ} \\
3 p^{3} \mathrm{P}_{0}-3 d^{3} \mathrm{D}_{1}^{\circ} \\
3 p^{1} \mathrm{~S}_{0}-5 d^{1} \mathrm{P}_{1}^{\circ}\end{array}$ \\
\hline $\begin{array}{l}2167.74 \\
2163.78 \\
2150.43 \\
2147.91 \\
2124.111\end{array}$ & $\begin{array}{c}7 \\
10 b \\
5 \\
3 \\
100 r\end{array}$ & & $\begin{array}{l}46116.47 \\
46200.86 \\
46487.64 \\
46542.18 \\
47063.58\end{array}$ & $\begin{array}{c}3 p^{1} \mathrm{~S}_{0}-6 d^{3} \mathrm{D}_{\mathrm{i}} \\
3 p{ }^{1} \mathrm{~S}_{0}-7 s^{3} \mathrm{P}_{1}^{\circ} \\
3 p{ }^{1} \mathrm{~S}_{0}-7 s^{1} \mathrm{P}_{1}^{\circ} \\
3 p p^{1} \mathrm{~S}_{0}-6 d^{3} \mathrm{P}_{1}^{\circ} \\
3 p^{1} \mathrm{D}_{2}-3 d^{1} \mathrm{~F}_{3}^{\circ}\end{array}$ \\
\hline $\begin{array}{l}2122.99 \\
2121.22 \\
2114.59 \\
2103.28 \\
2094.20\end{array}$ & $\begin{array}{c}10 \\
7 \\
4 b \\
5 b \\
2\end{array}$ & & $\begin{array}{l}47088.43 \\
47127.72 \\
47275.46 \\
47529.64 \\
47735.70\end{array}$ & $\begin{array}{l}3 p^{1}{ }^{1} \mathrm{D}_{2}-3 d^{1}{ }^{1} \mathrm{P}_{1} \\
3 p^{3} \mathrm{P}_{2}-3 d^{1} \mathrm{D}_{2}^{\circ} \\
3 p^{3} \mathrm{P}_{1}-3 d^{1} \mathrm{D}_{2}^{\circ} \\
3 p^{1} \mathrm{~S}_{0}-8 s^{3} \mathrm{P}_{2}^{\circ} \\
3 p^{1} \mathrm{~S}_{0}-8 s^{1} \mathrm{P}_{1}^{\circ}\end{array}$ \\
\hline $\begin{array}{l}2084.47 \\
2082.01 \\
2067.40 \\
2065.49 \\
2061.18\end{array}$ & $\begin{array}{r}20 \\
15 \\
1 \\
5 \\
8\end{array}$ & & $\begin{array}{l}47958.49 \\
48015.15 \\
48354.42 \\
48399.15 \\
48500.32\end{array}$ & $\begin{array}{l}3 p^{1} \mathrm{D}_{2}-4 d^{3} \mathrm{D}_{3}^{\circ} \\
3 p^{1} \mathrm{D}_{2}-5 s^{3} \mathrm{P}_{1}^{\circ} \\
3 p^{3} \mathrm{P}_{2}-3 p^{\prime}{ }^{3} \mathrm{D}_{2}^{\circ} \\
3 p^{3} \mathrm{P}_{0}-3 p^{\prime}{ }^{3} \mathrm{D}_{1}^{\circ} \\
3 p^{3} \mathrm{P}_{1}-3 p^{\prime}{ }^{3} \mathrm{D}_{2}^{\circ}\end{array}$ \\
\hline $\begin{array}{l}2058.13 \\
2054.81 \\
2010.97 \\
2008.43 \\
1991.23\end{array}$ & $\begin{array}{r}50 \\
8 \\
8 \\
3 \\
5\end{array}$ & & $\begin{array}{l}48572.18 \\
48650.65 \\
49711.10 \\
49773.96 \\
50203.82\end{array}$ & $\begin{array}{l}3 p^{1} \mathrm{D}_{2}-5 s^{1} \mathrm{P}_{1}^{\circ} \\
3 p^{3} \mathrm{P}_{2}-3 p^{\prime}{ }^{3} \mathrm{D}_{3}^{\circ} \\
3 p^{3} \mathrm{P}_{2}-3 d^{3} \mathrm{~F}_{3}^{\circ} \\
3 p^{3} \mathrm{P}_{1}-3 d^{3} \mathrm{~F}_{2}^{\circ} \\
3 p^{1} \mathrm{D}_{2}-4 d^{1} \mathrm{D}_{2}^{\circ}\end{array}$ \\
\hline $\begin{array}{l}1988.36 \\
1985.73 \\
1983.82 \\
1982.60 \\
1980.00\end{array}$ & $\begin{array}{r}30 \\
20 \\
3 \\
20 \\
10\end{array}$ & & $\begin{array}{l}50276.27 \\
50342.84 \\
50391.30 \\
50422.28 \\
50488.47\end{array}$ & $\begin{array}{c}3 p^{3} \mathrm{P}_{2}-3 d^{3} \mathrm{P}_{2} \\
3 p^{3} \mathrm{P}_{2}-3 d^{3} \mathrm{P}_{1} \\
3 p^{1} \mathrm{D}_{2}-4 d^{3} \mathrm{P}_{2}^{2} \\
3 p^{3} \mathrm{P}_{1}-3 d^{3} \mathrm{P}_{2} \\
3 p^{3} \mathrm{P}_{1}-3 d^{3} \mathrm{P}_{1}^{\circ}\end{array}$ \\
\hline $\begin{array}{l}1978.57 \\
1976.96\end{array}$ & $\begin{array}{l}12 \\
15\end{array}$ & & $\begin{array}{l}50524.95 \\
50566.08\end{array}$ & $\begin{array}{l}3 p^{3} \mathrm{P}_{1}-3 d^{3} \mathrm{P}_{0}^{\circ} \\
3 p^{3} \mathrm{P}_{0}-3 d^{3} \mathrm{P}_{1}^{\circ}\end{array}$ \\
\hline
\end{tabular}


TABLE 2.-Wave lengths in the arc spectrum of silicon emitted by the arc-innitrogen

\begin{tabular}{|c|c|c|c|}
\hline$\lambda_{\mathbf{v a c}} A$ & $\begin{array}{l}\text { Inten- } \\
\text { sity }\end{array}$ & $\nu_{\mathrm{Vac}} \mathrm{cm}^{-1}$ & Term combination \\
\hline $\begin{array}{l}\text { 1957. } 96 \\
1954.96 \\
1904.66 \\
1901.34 \\
1895.41\end{array}$ & $\begin{array}{r}0 \\
6 \\
12 \\
50 \\
2\end{array}$ & $\begin{array}{l}51021.44 \\
51151.94 \\
52502.80 \\
52594.48 \\
52759.03\end{array}$ & $\begin{array}{l}3 p^{1} \mathrm{D}_{2}-4 d^{3} \mathrm{~F}_{2}^{\circ} \\
3 p^{1} \mathrm{D}_{2}-4 d^{3} \mathrm{~F}_{3}^{\circ} \\
3 p^{1} \mathrm{D}_{2}-4 d^{1} \mathrm{P}_{1}^{\circ} \\
3 p^{1} \mathrm{D}_{2}-4 d^{1} \mathrm{~F}_{3}^{\circ} \\
3 p^{1} \mathrm{D}_{2}-5 d^{3} \mathrm{D}_{1}^{\circ}\end{array}$ \\
\hline $\begin{array}{l}\text { 1893. } 54 \\
1893.22 \\
1892.70 \\
1887.71 \\
1881.86\end{array}$ & $\begin{array}{r}4 \\
25 \\
3 \\
12 \\
12\end{array}$ & $\begin{array}{l}52811.13 \\
52820.06 \\
52834.57 \\
52974.24 \\
53138.92\end{array}$ & $\begin{array}{l}3 p^{1} \mathrm{D}_{2}-x^{\prime} \\
3 p^{1} \mathrm{D}_{2}-5 d^{3} \mathrm{D}_{3}^{\circ} \\
3 p p^{1} \mathrm{D}_{2}-x^{\prime \prime} \\
3 p^{1} \mathrm{D}_{2}-6 s^{3} \mathrm{P}_{1} \\
3 p^{3} \mathrm{P}_{2}-3 d^{1} \mathrm{~F}_{3}^{\circ}\end{array}$ \\
\hline $\begin{array}{l}\text { 1880. } 96 \\
1875.82 \\
1874.86 \\
1873.11 \\
1865.04\end{array}$ & $\begin{array}{r}5 \\
10 \\
25 \\
8 \\
2\end{array}$ & $\begin{array}{l}\text { 53164. } 34 \\
53310.02 \\
53337.32 \\
53387.15 \\
53618.15\end{array}$ & $\begin{array}{l}3 p^{3} \mathrm{P}_{2}-3 d^{1} \mathrm{P}_{1}^{\circ} \\
3 p^{3} \mathrm{P}_{1}-3 d^{1} \mathrm{P}_{1}^{\circ} \\
3 p^{1} \mathrm{D}_{2}-6 s^{1} \mathrm{P}_{1}^{\circ} \\
3 p^{3} \mathbf{P}_{0}-3 d^{1} \mathrm{P}_{1}^{\circ} \\
3 p^{1} \mathrm{D}_{2}-5 d^{3} \mathbf{P}_{2}^{\circ}\end{array}$ \\
\hline $\begin{array}{l}\text { 1861. } 80 \\
1853.17 \\
1852.48 \\
1851.80 \\
1850.68\end{array}$ & $\begin{array}{l}1 \\
10 \\
25 r \\
10 \\
50 r\end{array}$ & $\begin{array}{l}53711.46 \\
53961.60 \\
53981.69 \\
54001.51 \\
54034.19\end{array}$ & $\begin{array}{c}3 p p^{1} \mathrm{D}_{2}-5 d^{3} \mathrm{P}_{1}^{\circ} \\
3 p^{3} \mathrm{P}_{2}-4 d^{3} \mathrm{D}_{1}^{\circ} \\
3 p^{3} \mathrm{P}_{2}-4 d^{3} \mathrm{D}_{2}^{\circ} \\
3 p p^{1} \mathrm{D}_{2}-5 d^{1} \mathrm{D}_{2}^{\circ} \\
3 p^{3} \mathrm{P}_{2}-4 d^{3} \mathrm{D}_{3}^{\circ}\end{array}$ \\
\hline $\begin{array}{l}\text { 1848. } 75 \\
1848.16 \\
1847.47 \\
\text { 1846. } 13 \\
1845.53\end{array}$ & $\begin{array}{l}18 \\
20 r \\
35 r \\
12 \\
25 r\end{array}$ & $\begin{array}{l}54090.60 \\
54107.88 \\
54128.08 \\
54167.37 \\
54184.98\end{array}$ & $\begin{array}{l}3 p^{3} \mathrm{P}_{2}-5 s^{3} \mathrm{P}_{1}^{\circ} \\
3 p^{3} \mathrm{P}_{1}-4 d^{3} \mathrm{D}_{1}^{\circ} \\
3 p^{3} \mathrm{P}_{1}-4 d^{3} \mathrm{D}_{2}^{\circ} \\
3 p^{3} \mathrm{P}_{1}-5 s^{3} \mathrm{P}_{0}^{\circ} \\
3 p^{3} \mathrm{P}_{0}-4 d^{3} \mathrm{D}_{1}^{\circ}\end{array}$ \\
\hline $\begin{array}{l}\text { 1843. } 77 \\
1841.47 \\
1841.16 \\
1840.00 \\
1838.00\end{array}$ & $\begin{array}{l}15 \\
20 r \\
10 \\
2 \\
10\end{array}$ & $\begin{array}{l}54236.70 \\
54304.45 \\
54313.58 \\
54347.83 \\
54406.96\end{array}$ & $\begin{array}{l}3 p^{3} \mathrm{P}_{1}-5 s^{3} \mathrm{P}_{1}^{\circ} \\
3 p^{3} \mathrm{P}_{2}-5 s^{3} \mathrm{P}_{2}^{\circ} \\
3 p^{3} \mathrm{P}_{0}-5 s^{3} \mathrm{P}_{1}^{\circ} \\
3 p^{1} \mathrm{D}_{2}-5 d^{3} \mathrm{~F}_{2}^{\circ} \\
3 p^{1} \mathrm{D}_{2}-5 d^{3} \mathrm{~F}_{3}^{\circ}\end{array}$ \\
\hline $\begin{array}{l}\text { 1836. } 52 \\
1829.89 \\
1825.04 \\
1822.46 \\
1817.87\end{array}$ & $\begin{array}{r}20 \\
7 \\
1 \\
10 \\
2 b\end{array}$ & $\begin{array}{l}54450.81 \\
54648.09 \\
54793.32 \\
54870.90 \\
55009.44\end{array}$ & $\begin{array}{l}3 p^{3} \mathrm{P}_{1}-5 s^{3} \mathrm{P}_{2}^{\circ} \\
3 p^{3} \mathrm{P}_{2}-5 s^{1} \mathrm{P}_{1}^{\circ} \\
3 p^{3} \mathrm{P}_{1}-5 s^{1} \mathrm{P}_{1}^{\circ} \\
3 p^{3} \mathrm{P}_{0}-5 s^{1} \mathrm{P}_{1}^{\circ} \\
3 p^{1} \mathrm{D}_{2}-5 d^{1} \mathrm{P}_{1}^{\circ}\end{array}$ \\
\hline $\begin{array}{l}1814.09 \\
1814.02 \\
1809.56 \\
1809.05 \\
1808.48\end{array}$ & $\begin{array}{l}30 \\
50 \\
3 b \\
30 \\
4 b\end{array}$ & $\begin{array}{l}55124.05 \\
55126.18 \\
55262.06 \\
55277.64 \\
55295.05\end{array}$ & $\begin{array}{l}3 p{ }^{1} \mathrm{D}_{2}-6 d^{3} \mathrm{D}_{2}^{\circ} \\
3 p^{1} \mathrm{D}_{2}-5 d^{1} \mathrm{~F}_{3}^{\circ} \\
\\
3 p{ }^{1} \mathrm{D}_{2}-6 d^{3} \mathrm{D}^{\circ} \\
3 p^{1} \mathrm{D}_{2}-7 s^{3} \mathrm{P}_{1}^{\circ}\end{array}$ \\
\hline $\begin{array}{l}\text { 1799. } 14 \\
1797.33 \\
1790.28 \\
1784.11 \\
1783.23\end{array}$ & $\begin{array}{r}10 \\
3 \\
4 \\
1 \\
8\end{array}$ & $\begin{array}{l}55582.11 \\
55638.18 \\
55857.19 \\
56050.36 \\
56078.02\end{array}$ & $\begin{array}{l}3 p^{1} \mathrm{D}_{2}-7 s^{1} \mathrm{P}_{1}^{\circ} \\
3 p{ }^{1} \mathrm{D}_{2}-6 d^{3} \mathrm{P}_{1}^{\circ} \\
3 p p^{1} \mathrm{D}_{2}-6 d^{1} \mathrm{D}_{2}^{o} \\
3 p p^{1} \mathrm{D}_{2}-6 d^{3} \mathrm{~F}_{2}^{\circ} \\
3 p^{1} \mathrm{D}_{2}-6 d^{3} \mathrm{~F}_{3}^{\circ}\end{array}$ \\
\hline $\begin{array}{l}1776.85 \\
1774.96 \\
1772.24 \\
1770.94 \\
1770.63\end{array}$ & $\begin{array}{c}10 \\
0 e \\
1 \\
10 \\
8\end{array}$ & $\begin{array}{l}56279.36 \\
56339.29 \\
56425.76 \\
56467.18 \\
56477.07\end{array}$ & $\begin{array}{l}3 p^{3} \mathrm{P}_{2}-4 d^{1} \mathrm{D}_{2}^{\circ} \\
3 p^{3} \mathrm{P}_{1}-4 d d^{1} \mathrm{D}_{2}^{\circ} \\
3 p{ }^{3} \mathrm{P}_{2}-4 d^{3} \mathrm{P}_{2}^{\circ} \\
3 p^{3} \mathrm{P}_{2}-4 d^{3} \mathrm{P}_{1}^{\circ}\end{array}$ \\
\hline
\end{tabular}


TABLE 2.-Wave lengths in the arc spectrum of silicon emitted by the arc-innitrogen-Continued

\begin{tabular}{|c|c|c|c|}
\hline$\lambda_{\mathrm{vac}} \mathrm{A}$ & $\begin{array}{l}\text { Inten- } \\
\text { sity }\end{array}$ & $\nu_{\mathrm{vac}} \mathrm{cm}^{-1}$ & Term combination \\
\hline $\begin{array}{l}1769.78 \\
1769.60 \\
1766.34\end{array}$ & $\begin{array}{r}15 \\
1 \\
5\end{array}$ & $\begin{array}{l}56504.20 \\
56509.95 \\
56614.24\end{array}$ & $\begin{array}{l}3 p^{1} \mathrm{D}_{2}-6 d^{1} \mathrm{~F}_{3}^{\circ} \\
3 p^{1} \mathrm{D}_{2}-8 s^{3} \mathrm{P}_{1}^{\circ} \\
3 p^{3} \mathrm{P}_{1}-4 d^{3} \mathrm{P}_{2}^{\circ}\end{array}$ \\
\hline 1766. 03 & 6 & 56624. 18 & $\begin{array}{l}3 p^{1} \mathrm{D}_{2}-8 s^{3} \mathrm{P}_{2}^{\circ} \\
3 p^{3} \mathrm{P}_{1}-4 d^{3} \mathrm{P}_{i}\end{array}$ \\
\hline 1765.82 & 2 & 56630. 92 & $3 p^{1} \mathrm{D}_{2}-6 d^{1} \mathrm{P}_{1}^{1}$ \\
\hline $\begin{array}{l}1765.61 \\
1765.02 \\
1763.67 \\
1759.56 \\
1753.13\end{array}$ & $\begin{array}{l}5 b \\
5 \\
4 \\
3 \\
4 b\end{array}$ & $\begin{array}{l}56637.65 \\
56656.58 \\
56699.96 \\
56832.39 \\
57040.83\end{array}$ & $\begin{array}{c}3 p^{1} \mathrm{D}_{2}-7 d^{3} \mathrm{D}_{3}^{\circ} \\
3 p^{3} \mathrm{P}_{1}-4 d^{3} \mathrm{P}_{0}^{0} \\
3 p^{3} \mathrm{P}_{0}-4 d^{3} \mathrm{P}_{1}^{\circ} \\
3 p^{1} \mathrm{D}_{2}-8 s^{1} \mathrm{P}_{1}^{\circ} \\
3 p^{1} \mathrm{D}_{2}-7 d^{1} \mathrm{D}_{2}^{\circ}\end{array}$ \\
\hline $\begin{array}{l}\text { 1752. } 68 \\
1749.74 \\
1747.36 \\
1745.35 \\
1743.88\end{array}$ & $\begin{array}{l}0 \\
0 \\
4 \\
2 \\
5 b\end{array}$ & $\begin{array}{l}57055.48 \\
57151.34 \\
57229.20 \\
57295.10 \\
57343.40\end{array}$ & $\begin{array}{l}3 p^{1} \mathrm{D}_{2}-7 d^{3} \mathrm{~F}_{3}^{\circ} \\
3 p^{3} \mathrm{P}_{2}-4 d^{3} \mathrm{~F}_{2}^{\circ} \\
3 p^{3} \mathrm{P}_{2}-4 d^{3} \mathrm{~F}_{3}^{\circ} \\
3 p^{3} \mathrm{P}_{1}-4 d^{3} \mathrm{~F}_{2}^{\circ} \\
3 p^{1} \mathrm{D}_{2}-7 d^{1} \mathrm{~F}_{3}^{\circ}\end{array}$ \\
\hline $\begin{array}{l}1740.34 \\
1736.50 \\
1734.70 \\
1707.09 \\
1704.44\end{array}$ & $\begin{array}{l}3 h \\
1 \\
3 \\
0 \\
7 r\end{array}$ & $\begin{array}{l}57460.04 \\
57587.10 \\
57646.86 \\
58579.22 \\
58670.29\end{array}$ & $\begin{array}{l}3 p^{1} \mathrm{D}_{2}-8 d^{3} \mathrm{D}_{3}^{\circ} \\
3 p^{1} \mathrm{D}_{2}-9 s^{1} \mathrm{P}_{1}^{0} \\
\\
3 p^{3} \mathrm{P}_{2}-4 d^{1} \mathrm{P}_{1}^{\circ} \\
3 p^{3} \mathrm{P}_{2}-4 d^{1} \mathrm{~F}_{3}^{\circ}\end{array}$ \\
\hline $\begin{array}{l}1702.81 \\
1700.60 \\
1700.43 \\
1699.70 \\
1698.18\end{array}$ & $\begin{array}{c}5 \\
4 \\
15 r \\
1 \\
4 b\end{array}$ & $\begin{array}{l}58726.46 \\
58802.78 \\
58808.65 \\
58833.91 \\
58886.58\end{array}$ & $\begin{array}{l}3 p^{3} \mathrm{P}_{1}-4 d^{1} \mathrm{P}_{1} \\
3 p^{3} \mathrm{P}_{0}-4 d^{1} \mathrm{P}_{1}^{\circ} \\
3 p^{3} \mathrm{P}_{2}-5 d^{3} \mathrm{D}_{2}^{\circ} \\
3 p^{3} \mathrm{P}_{2}-5 d^{3} \mathrm{D}_{1}^{\circ} \\
3 p^{3} \mathrm{P}_{2}-x^{\prime}\end{array}$ \\
\hline $\begin{array}{l}\text { 1697. } 96 \\
1697.55 \\
1696.20 \\
1695.50 \\
1693.47\end{array}$ & $\begin{array}{c}20 r \\
3 b \\
20 r \\
5 r \\
5\end{array}$ & $\begin{array}{l}58894.19 \\
58908.43 \\
58955.31 \\
58979.65 \\
59050.35\end{array}$ & $\begin{array}{l}3 p^{3} \mathrm{P}_{2}-5 d^{3} \mathrm{D}_{3}^{\circ} \\
3 p^{3} \mathrm{P}_{2}-x^{\prime \prime} \\
3 p^{3} \mathrm{P}_{1}-5 d^{3} \mathrm{D}_{2}^{\circ} \\
3 p^{3} \mathrm{P}_{1}-5 d^{3} \mathrm{D}_{1}^{\circ} \\
3 p^{3} \mathrm{P}_{2}-6 s^{3} \mathrm{P}_{1}^{\circ}\end{array}$ \\
\hline $\begin{array}{l}1693.30 \\
1690.77 \\
1689.28 \\
1687.06 \\
1686.83\end{array}$ & $\begin{array}{l}7 r \\
3 \\
3 \\
1 \\
4\end{array}$ & $\begin{array}{l}59056.28 \\
59144.65 \\
59196.82 \\
59274.71 \\
59282.80\end{array}$ & $\begin{array}{l}3 p^{3} \mathrm{P}_{0}-5 d^{3} \mathrm{D}_{1}^{\circ} \\
3 p^{3} \mathrm{P}_{1}-6 s^{3} \mathrm{P}_{0}^{\circ} \\
3 p^{3} \mathrm{P}_{1}-6 s^{3} \mathrm{P}_{1}^{\circ} \\
3 p^{3} \mathrm{P}_{0}-6 s^{3} \mathrm{P}_{1}^{\circ} \\
3 p^{3} \mathrm{P}_{2}-6 s^{3} \mathrm{P}_{2}^{\circ}\end{array}$ \\
\hline $\begin{array}{l}1682.67 \\
1676.80 \\
1675.23 \\
1672.60 \\
1671.11\end{array}$ & $\begin{array}{l}3 \\
1 \\
4 r \\
3 \\
2\end{array}$ & $\begin{array}{l}\text { 59429. } 36 \\
59637.40 \\
59693.30 \\
\text { 59787. } 16 \\
\text { 59840. } 46\end{array}$ & $\begin{array}{l}3 p^{3} \mathrm{P}_{1}-6 s^{3} \mathrm{P}_{2}^{\circ} \\
3 p^{3} \mathrm{P}_{0}-6 s^{1} \mathrm{P}_{1}^{\circ} \\
3 p^{3} \mathrm{P}_{2}-5 d^{3} \mathrm{P}_{2}^{0} \\
3 p^{3} \mathrm{P}_{2}-5 d^{3} \mathrm{P}_{1}^{\circ} \\
3 p^{3} \mathrm{P}_{1}-5 d^{3} \mathrm{P}_{2}^{\circ}\end{array}$ \\
\hline $\begin{array}{l}1668.52 \\
1667.63 \\
1666.36 \\
1664.54 \\
1660.47\end{array}$ & $\begin{array}{l}3 \\
3 \\
2 \\
2 \\
0\end{array}$ & $\begin{array}{l}59933.35 \\
59965.34 \\
60011.04 \\
60076.66 \\
60223.91\end{array}$ & $\begin{array}{l}3 p^{3} \mathrm{P}_{1}-5 d^{3} \mathrm{P}_{1}^{\circ} \\
3 p^{3} \mathrm{P}_{1}-5 d^{3} \mathrm{P}_{0}^{\circ} \\
3 p^{3} \mathrm{P}_{0}-5 d^{3} \mathrm{P}_{1}^{\circ} \\
3 p^{3} \mathrm{P}_{2}-5 d^{1} \mathrm{D}_{2}^{\circ} \\
3 p^{3} \mathrm{P}_{1}-5 d^{1} \mathrm{D}_{2}^{\circ}\end{array}$ \\
\hline
\end{tabular}


$\mathrm{T}_{\mathrm{AB}} \mathrm{LE}$ 2.-Wave lengths in the arc spectrum of silicon emitted by the arc-innitrogen-Continued

\begin{tabular}{|c|c|c|c|}
\hline$\lambda_{\mathrm{vac}} \mathrm{A}$ & $\begin{array}{l}\text { Inten- } \\
\text { sity }\end{array}$ & $\nu_{\mathrm{vac}} \mathrm{cm}^{-1}$ & Term combination \\
\hline $\begin{array}{l}\text { 1653. } 36 \\
1651.05 \\
1633.99 \\
1633.90 \\
1633.15\end{array}$ & $\begin{array}{l}1 \\
0 \\
3 \\
4 \\
2 r\end{array}$ & $\begin{array}{l}60482.89 \\
60567.52 \\
61199.88 \\
61203.26 \\
61231.36\end{array}$ & $\begin{array}{l}3 p^{3} \mathrm{P}_{2}-5 d^{3} \mathrm{~F}_{3}^{\circ} \\
3 p^{3} \mathrm{P}_{1}-5 d^{3} \mathrm{~F}_{2}^{\circ} \\
3 p^{3} \mathrm{P}_{2}-6 d^{3} \mathrm{D}_{2}^{\circ} \\
3 p^{3} \mathrm{P}_{2}-5 d^{1} \mathrm{~F}_{3}^{\circ} \\
3 p^{3} \mathrm{P}_{1}-5 d^{1} \mathrm{P}_{1}^{\circ}\end{array}$ \\
\hline $\begin{array}{l}1631.11 \\
1630.15 \\
1629.96 \\
1629.47 \\
1627.70\end{array}$ & $\begin{array}{l}4 r \\
7 r \\
8 r \\
7 r \\
2\end{array}$ & $\begin{array}{l}61307.94 \\
61344.05 \\
61351.19 \\
61369.65 \\
61436.38\end{array}$ & $\begin{array}{l}3 p^{3} \mathrm{P}_{0}-5 d^{1} \mathrm{P}_{1} \\
3 p^{3} \mathrm{P}_{1}-6 d^{3} \mathrm{D}_{2} \\
3 p{ }^{3} \mathrm{P}_{2}-6 d^{3} \mathrm{D}_{3} \\
3 p^{3} \mathrm{P}_{2}-7 s^{3} \mathrm{P}_{1}^{\circ} \\
3 p^{3} \mathrm{P}_{1}-6 d^{3} \mathrm{D}_{1}^{\circ}\end{array}$ \\
\hline $\begin{array}{l}1627.03 \\
1625.71 \\
1625.58 \\
1623.34 \\
1622.87\end{array}$ & $\begin{array}{l}1 \\
5 r \\
1 \\
0 \\
4 r\end{array}$ & $\begin{array}{l}61461.68 \\
61511.58 \\
61516.51 \\
61601.40 \\
61619.23\end{array}$ & $\begin{array}{l}3 p^{3} \mathrm{P}_{1}-7 s^{3} \mathrm{P}_{0}^{\circ} \\
3 p^{3} \mathrm{P}_{0}-6 d^{3} \mathrm{D}_{1}^{\circ} \\
3 p^{3} \mathrm{P}_{1}-7 s^{3} \mathrm{P}_{1}^{\circ} \\
3 p^{3} \mathrm{P}_{2}-7 s^{3} \mathrm{P}_{2}^{\circ} \\
3 p^{3} \mathrm{P}_{2}-6 d^{3} \mathrm{P}_{2}^{\circ}\end{array}$ \\
\hline $\begin{array}{l}1620.39 \\
1619.53 \\
1619.00 \\
1616.55 \\
1615.89\end{array}$ & $\begin{array}{l}3 \\
1 \\
0 \\
3 \\
3\end{array}$ & $\begin{array}{l}61713.54 \\
61746.32 \\
61766.52 \\
61860.14 \\
61885.40\end{array}$ & $\begin{array}{l}3 p^{3} \mathrm{P}_{2}-6 d^{3} \mathrm{P}_{1}^{\circ} \\
3 p^{3} \mathrm{P}_{1}-7 s^{3} \mathrm{P}_{2}^{\circ} \\
3 p^{3} \mathrm{P}_{1}-6 d^{3} \mathrm{P}_{2}^{\circ} \\
3 p^{3} \mathrm{P}_{1}-6 d^{3} \mathrm{P}_{1}^{\circ} \\
3 p^{3} \mathrm{P}_{0}-7 s^{1} \mathrm{P}_{1}^{\circ}\end{array}$ \\
\hline $\begin{array}{l}1614.60 \\
1614.55 \\
1608.92 \\
1605.87 \\
1597.99\end{array}$ & $\begin{array}{l}1 \\
3 \\
2 \\
1 \\
3\end{array}$ & $\begin{array}{l}61934.84 \\
61936.76 \\
62153.50 \\
62271.55 \\
62578.60\end{array}$ & $\begin{array}{l}3 p^{3} \mathrm{P}_{2}-6 d^{1} \mathrm{D}_{2}^{2} \\
3 p^{3} \mathrm{P}_{0}-6 d^{3} \mathrm{P}_{1} \\
3 p^{3} \mathrm{P}_{2}-6 d^{3} \mathrm{~F}_{3}^{3} \\
3 p^{3} \mathrm{P}_{1}-6 d^{3} \mathrm{~F}_{2}^{2} \\
3 p^{3} \mathrm{P}_{2}-6 d^{1} \mathrm{~F}_{3}^{3}\end{array}$ \\
\hline $\begin{array}{l}1597.83 \\
1595.82 \\
1595.50 \\
1594.92 \\
1594.53\end{array}$ & $\begin{array}{l}2 \\
1 \\
1 \\
4 r \\
5 r\end{array}$ & $\begin{array}{l}62584.88 \\
62663.70 \\
62676.28 \\
62699.07 \\
62714.40\end{array}$ & $\begin{array}{l}3 p^{3} \mathrm{P}_{2}-8 s^{3} \mathrm{P}_{1}^{\circ} \\
3 p^{3} \mathrm{P}_{2}-7 d^{3} \mathrm{D}_{2} \\
3 p^{3} \mathrm{P}_{1}-8 s^{3} \mathrm{P}_{0}^{\circ} \\
3 p^{3} \mathrm{P}_{2}-8 s^{3} \mathrm{P}_{2}^{\circ} \\
3 p^{3} \mathrm{P}_{2}-7 d^{3} \mathrm{D}_{3}^{\circ}\end{array}$ \\
\hline $\begin{array}{l}1592.45 \\
1592.35 \\
1592.15 \\
1591.17 \\
1590.49\end{array}$ & $\begin{array}{l}1 \\
3 r \\
3 r \\
0 \\
2 r\end{array}$ & $\begin{array}{l}62796.32 \\
62800.24 \\
62808.12 \\
62846.82 \\
62873.70\end{array}$ & $\begin{array}{l}3 p^{3} \mathrm{P}_{1}-7 d^{3} \mathrm{D}_{1}^{\circ} \\
3 p^{3} \mathrm{P}_{1}-7 d^{3} \mathrm{D}_{2} \\
3 p^{3} \mathrm{P}_{0}-8 s^{3} \mathrm{P}_{1}^{\circ} \\
3 p^{3} \mathrm{P}_{1}-8 s^{3} \mathrm{P}_{2}^{\circ} \\
3 p^{3} \mathrm{P}_{0}-7 d^{3} \mathrm{D}_{1}^{\circ}\end{array}$ \\
\hline $\begin{array}{l}1589.60 \\
1586.76 \\
1586.00 \\
1584.27\end{array}$ & $\begin{array}{l}1 \\
1 \\
1 \\
0\end{array}$ & $\begin{array}{l}62908.90 \\
63021.51 \\
63051.70 \\
63120.55\end{array}$ & $\begin{array}{l}3 p^{3} \mathrm{P}_{2}-8 s^{1} \mathrm{P}_{1}^{\circ} \\
3 p^{3} \mathrm{P}_{1}-8 s^{1} \mathrm{P}_{1}^{\circ} \\
3 p^{3} \mathrm{P}_{2}-7 d^{1} \mathrm{D}_{2}^{\circ}\end{array}$ \\
\hline 1583.97 & 0 & 63132.50 & $\begin{array}{l}3 p^{3} \mathrm{P}_{0}-8 s^{1} \mathrm{P}_{1} \\
3 p^{3} \mathrm{P}_{2}-7 d^{3} \mathrm{~F}_{3}^{0}\end{array}$ \\
\hline $\begin{array}{l}1580.28 \\
1576.76 \\
1574.69 \\
1573.85 \\
1565.30\end{array}$ & $\begin{array}{l}0 \\
1 \\
2 \\
1 r \\
1\end{array}$ & $\begin{array}{l}63279.92 \\
63421.20 \\
63504.56 \\
63538.47 \\
63885.52\end{array}$ & $\begin{array}{l}3 p^{3} \mathrm{P}_{2}-7 d^{1} \mathrm{~F}_{3}^{\circ} \\
3 p^{3} \mathrm{P}_{2}-8 d^{3} \mathrm{D}_{3}^{\circ} \\
3 p^{3} \mathrm{P}_{0}-9 s^{1} \mathrm{P}_{1}^{\circ}\end{array}$ \\
\hline
\end{tabular}


The intensities assigned to the lines are the usual visual estimates, and are not comparable between widely separated regions of the spectrum. The letters following the estimated intensities give a description of the line as emitted by the arc in air, even though for all the lines between 7970 and $6124 \mathrm{~A}$, except a few fainter ones, the recorded wave lengths are for the enclosed arc at reduced pressure.

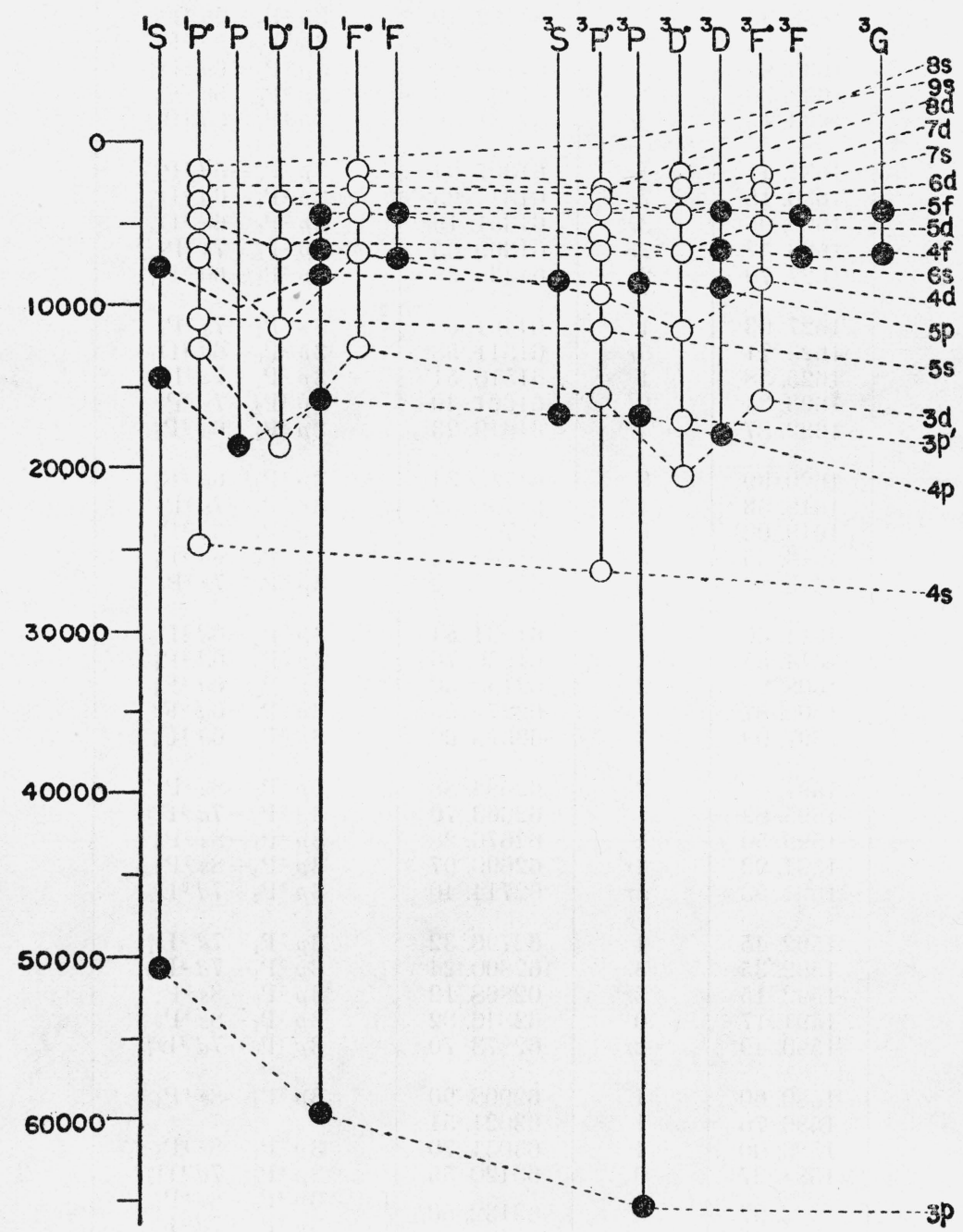

Figure 1.-Terms of the arc spectrum of silicon; even terms; $\bigcirc$ odd terms.

These letters have the following significance: $b$, widened, not sharp; $e$, enhanced at electrode; $h$, hazy, $H$, very hazy; $l$, unsymmetrically shaded toward red; $r$, reversed.

\section{TERM STRUCTURE OF SI I}

The terms upon which the arc spectrum of silicon is built result from the interactions of a migrating valence electron with the con- 
figurations $3 s^{2} 3 p$ and $3 s 3 p^{2}$. The terms to be expected theoretically from this procedure are set forth in table 3 . Those that have actually been established in the analysis of the spectrum, both through Fowler's work and the work at the National Bureau of Standards, are listed in table 4, of which figure 1 is a graphic representation.

TABLE 3.-Theoretical terms of Si I

\begin{tabular}{|l|l|}
\hline $\begin{array}{c}\text { Electron con- } \\
\text { figuration }\end{array}$ & \multicolumn{1}{|c|}{ Terms } \\
\hline & \\
$3 s^{2} 3 p^{2}$ & ${ }^{3} \mathrm{P},{ }^{1} \mathrm{D},{ }^{1} \mathrm{~S}$ \\
$3 s^{2} 3 p n s$ & ${ }^{3} \mathrm{P}^{\circ},{ }^{1} \mathrm{P}^{\circ}$ \\
$3 s^{2} 3 p n p$ & ${ }^{2} \mathrm{D},{ }^{3} \mathrm{P},{ }^{3} \mathrm{~S},{ }^{1} \mathrm{D},{ }^{1} \mathrm{P},{ }^{1} \mathrm{~S}$ \\
$3 s^{2} 3 p n d$ & ${ }^{3} \mathrm{~F}^{\circ},{ }^{3} \mathrm{D}^{\circ},{ }^{3} \mathrm{P}^{\circ},{ }^{\circ} \mathrm{F}^{\circ},{ }^{1} \mathrm{D}^{\circ},{ }^{\circ} \mathrm{P}^{\circ}$ \\
$3 s^{2} 3 p n f$ & ${ }^{3} \mathrm{GG}^{3}{ }^{3},{ }^{3} \mathrm{D},{ }^{1} \mathrm{G},{ }^{1} \mathrm{~F},{ }^{1} \mathrm{D}$ \\
$3 s 3 p^{3}=3 p^{\prime}$ & ${ }^{5} \mathrm{~S}^{\circ},{ }^{3} \mathrm{D}^{\circ},{ }^{3} \mathrm{P}^{\circ},{ }^{3} \mathrm{~S}^{\circ},{ }^{1} \mathrm{D}^{\circ},{ }^{1} \mathrm{P}^{\circ}$ \\
&
\end{tabular}

TABLE 4.-Observed terms of Si I.

\begin{tabular}{|c|c|c|c|c|c|}
\hline $\begin{array}{c}\text { Term } \\
\text { symbol }\end{array}$ & $\begin{array}{l}\text { Term } \\
\text { value } \\
\mathrm{cm}^{-1}\end{array}$ & $\triangle \nu$ & $\begin{array}{c}\text { Term } \\
\text { symbol }\end{array}$ & $\begin{array}{l}\text { Term } \\
\text { value } \\
\mathrm{cm}^{-1}\end{array}$ & $\triangle \nu$ \\
\hline $3 p^{3} \mathrm{P}_{0}$ & 65743.00 & \multirow{5}{*}{$\begin{array}{r}77.15 \\
146.16 \\
6075.50 \\
9095.43\end{array}$} & $3 d^{1} \mathrm{D}_{2}^{\circ}$ & 18391. 50 & \multirow{5}{*}{$\begin{array}{l}178.45 \\
296.36\end{array}$} \\
\hline $3 p^{3} \mathrm{P}_{1}$ & 65665.85 & & $\begin{array}{l}3 d^{1} \mathrm{~F}_{3} \\
3 d^{1} \mathrm{P}_{1}^{\circ}\end{array}$ & $\begin{array}{l}12380.59 \\
12355.83\end{array}$ & \\
\hline $3 p^{3} \mathrm{P}_{2}$ & 65519.69 & & $3 p^{\prime}{ }^{3} \mathrm{D}_{\mathrm{i}}$ & 17343.85 & \\
\hline $3 p{ }^{1} \mathrm{D}_{2}$ & 59444. 19 & & $3 p^{\prime}{ }^{3} \mathrm{D}_{2}^{\circ}$ & 17165.40 & \\
\hline $3 p^{1} \mathrm{~S}_{0}$ & 50348. 76 & & $3 p^{\prime}{ }^{3} \mathrm{D}_{3}^{\circ}$ & 16869.04 & \\
\hline $4 s^{3} \mathrm{P}_{0}^{\circ}$ & 26059. 90 & \multirow{4}{*}{$\begin{array}{r}77.10 \\
194.92 \\
1036.62\end{array}$} & $4 p^{3} \mathrm{D}_{1}$ & 17723.00 & \multirow{3}{*}{$\begin{array}{r}82.38 \\
161.97\end{array}$} \\
\hline $4 s^{3} \mathrm{P}_{\mathrm{i}}$ & 25982. 80 & & $4 p^{3} \mathrm{D}_{2}$ & 17640.62 & \\
\hline $4 s^{3} \mathrm{P}_{2}^{\circ}$ & 25787. 88 & & $4 p^{3} \mathrm{D}_{3}$ & 17478.65 & \\
\hline $4 s^{1} \mathrm{P}_{\mathrm{i}}$ & 24751. 26 & & $4 p^{3} \mathrm{P}_{0}$ & 16714. 83 & \multirow{3}{*}{$\begin{array}{r}32.38 \\
128.06\end{array}$} \\
\hline $3 d^{3} \mathrm{D}_{1}^{\circ}$ & 20466.80 & \multirow{3}{*}{$\begin{array}{l}\text { 17. } 40 \\
\text { 28. } 26\end{array}$} & $4 p^{3} \mathrm{P}_{1}$ & 16682.45 & \\
\hline $3 d^{3} \mathrm{D}_{2}^{\circ}$ & 20449. 40 & & $4 p^{3} \mathrm{P}_{2}$ & 16554. 39 & \\
\hline $3 d^{3} \mathrm{D}_{3}^{\circ}$ & 20421. 14 & & $4 p^{3} \mathrm{~S}_{1}$ & 16343. 34 & \multirow{7}{*}{$\begin{array}{r}69.32 \\
213.98 \\
\text { 343. } 11\end{array}$} \\
\hline $3 d^{3} \mathrm{~F}_{2}^{\circ}$ & 15892.07 & \multirow{3}{*}{$\begin{array}{r}83.19 \\
137.76\end{array}$} & $\begin{array}{l}4 p^{1}{ }^{1} \mathrm{P}_{1} \\
4 p^{1} \mathrm{D}_{3}\end{array}$ & $\begin{array}{l}18458.80 \\
15553.57\end{array}$ & \\
\hline $3 d^{3} \mathrm{~F}_{3}^{\circ}$ & 15808.88 & & $4 p^{1} \mathrm{~S}_{0}$ & 14131. 23 & \\
\hline $3 d^{3} \mathrm{~F}_{4}^{\circ}$ & 15671. 12 & & $5 s^{3} \mathrm{P}_{0}^{\circ}$ & 11498.42 & \\
\hline $3 d^{3} \mathrm{P}_{2}^{\circ}$ & 15243.56 & \multirow{3}{*}{$\begin{array}{l}-66.51 \\
-36.20\end{array}$} & $5 s^{3} \mathrm{P}_{1}$ & 11429.10 & \\
\hline $3 d^{3} \mathrm{P}_{1}$ & 15177.05 & & $5 s^{3} \mathrm{P}_{2}^{\circ}$ & 11215. 12 & \\
\hline $3 d^{3} \mathrm{P}_{0}^{\circ}$ & 15140.85 & & $5 s^{1} \mathrm{P}_{1}$ & 10872.01 & \\
\hline
\end{tabular}


TABLE 4.-Observed terms of Si I.-Continued

\begin{tabular}{|c|c|c|c|c|c|}
\hline $\begin{array}{c}\text { Term } \\
\text { symbol }\end{array}$ & $\begin{array}{l}\text { Term } \\
\text { value } \\
\mathrm{cm}^{-1}\end{array}$ & $\triangle \nu$ & $\begin{array}{c}\text { Term } \\
\text { symbol }\end{array}$ & $\begin{array}{l}\text { Term } \\
\text { value } \\
\mathrm{cm}^{-1}\end{array}$ & $\triangle \nu$ \\
\hline & & & $4 f^{1} \mathrm{~F}_{3}$ & 6968.82 & \\
\hline $4 d^{3} \mathrm{D}_{\mathrm{i}}^{\circ}$ & 11558. 03 & & $4 f^{1} \mathrm{G}_{4}$ & & \\
\hline $4 d^{3} \mathrm{D}_{?}$ & 11537,88 & 20. 15 & $4 f^{1} \mathrm{D}_{2}$ & 6632. 09 & \\
\hline & & 52. 28 & $x^{\prime}$ & 6633. 1 & 22. 6 \\
\hline & & & $x^{\prime \prime}$ & 6610.5 & \\
\hline $4 d^{3} \mathrm{P}_{\mathrm{i}}$ & 9042.16 & -9.90 & $6 s^{3} \mathrm{P}_{0}^{\circ}$ & 6522.24 & 52. 52 \\
\hline $4 d^{3} \mathrm{P}_{0}^{\circ}$ & 9009.76 & -32.40 & $6 s^{3} \mathrm{P}_{1}^{\circ}$ & 6469. 72 & 232. 89 \\
\hline $4 d^{3} \mathrm{~F}_{2}^{\circ}$ & 8370.56 & & $6 s^{3} P_{2}^{\circ}$ & 6236.83 & 130. 17 \\
\hline $4 d^{3} \mathrm{~F}_{3}^{\circ}$ & 8292.30 & 78. 26 & $6 s^{1} \mathrm{P}_{1}^{\circ}$ & 6106.66 & \\
\hline $4 d^{3} \mathrm{~F}_{4}^{\circ}$ & 8159.15 & 133.15 & $5 d^{3} \mathrm{D}_{1}^{\circ}$ & $\begin{array}{l}6686.30 \\
6710.58\end{array}$ & -24.28 \\
\hline $\begin{array}{l}4 d^{1} \mathrm{D}_{2}^{\circ} \\
4 d^{1} \mathrm{P}_{1}^{\circ} \\
4 d^{1} \mathrm{~F}_{3}^{\circ}\end{array}$ & $\begin{array}{l}9240.00 \\
6941.00 \\
6849.72\end{array}$ & & $5 d^{3} \mathrm{D}_{3}^{\circ}$ & 6624. 49 & 86. 09 \\
\hline $5 p^{3} \mathrm{D}_{1}$ & 8765.00 & & $5 d^{3} \mathrm{P}_{2}^{\circ}$ & & -92.75 \\
\hline $5 p^{3} \mathrm{D}_{2}$ & 8725.74 & $\begin{array}{r}39.26 \\
180.68\end{array}$ & $\begin{array}{l}5 d^{3} \mathrm{P}_{1}^{\circ} \\
5 d^{3} \mathrm{P}_{0}^{\circ}\end{array}$ & $\begin{array}{l}5732.90 \\
5700.52\end{array}$ & -32.38 \\
\hline $5 p^{3} \mathrm{D}_{3}$ & 8545.06 & & $5 d^{3} \mathrm{~F}_{2}^{\circ}$ & 5097. 51 & \\
\hline $5 p^{3} \mathrm{P}_{0}$ & 8447. 24 & 32.88 & $5 d^{3} \mathrm{~F}_{3}^{\circ}$ & 5037.10 & 60.41 \\
\hline $5 p^{3} \mathrm{P}_{1}$ & 8414. 36 & 139.54 & $5 d^{3} \mathrm{~F}_{9}^{\circ}$ & 4893.87 & 143. 23 \\
\hline $5 p^{3} \mathrm{P}_{2}$ & 8274. 82 & & & 5443. 08 & \\
\hline $5 p{ }^{3} \mathrm{~S}_{1}$ & 8201.14 & & $\begin{array}{l}5 d^{1} \mathrm{P}_{1}^{2} \\
5 d^{1} \mathrm{~F}_{3}^{\circ}\end{array}$ & $\begin{array}{l}4434.68 \\
4319.00\end{array}$ & \\
\hline $\begin{array}{l}5 p{ }^{1} \mathrm{D}_{2} \\
5 p{ }^{1} \mathrm{P}_{1} \\
5 p \\
{ }^{1} \mathrm{~S}_{0}\end{array}$ & $\begin{array}{l}7945.18 \\
9317.9 ? \\
7431.81\end{array}$ & & $5 f^{3} \mathrm{~F}_{2}$ & 4438. 50 & 0. 36 \\
\hline $4 f^{3} \mathrm{~F}_{2}$ & 6967.56 & 1136 & $5 f^{3} \mathrm{~F}_{3}$ & 4438. 14 & 1. 71 \\
\hline $4 f^{3} \mathrm{~F}_{3}$ & 6956. 20 & $\begin{array}{r}11.00 \\
2.20\end{array}$ & $5 f^{3} \mathrm{G}_{3}$ & $\begin{array}{l}4436.43 \\
4180.63\end{array}$ & \\
\hline $4 f^{3} \mathrm{~F}_{4}$ & 6954.00 & & $5 f^{3} \mathrm{G}_{4}$ & $\begin{array}{r}4100.00 \\
4179.25\end{array}$ & 1. 38 \\
\hline $4 f^{3} \mathrm{G}_{3}$ & 6707.85 & 185 & $5 f^{3} \mathrm{G}_{5}$ & & \\
\hline $4 f^{3} \mathrm{G}_{4}$ & 6706.00 & 1684 & $5 f^{3} \mathrm{D}_{3}$ & 4145. 88 & -078 \\
\hline $4 f^{3} \mathrm{G}_{5}$ & 6689. 16 & & $5 f^{3} \mathrm{D}_{2}$ & 4145. 10 & \\
\hline $4 f^{3} \mathrm{D}_{3}$ & 6633. 25 & -8109 & $5 f^{3} \mathrm{D}_{1}$ & 4144. 40 & \\
\hline $4 f^{3} \mathrm{D}_{2}$ & 6552. 16 & 0.44 & $\begin{array}{l}5 f^{1} \mathrm{~F}_{3} \\
5 f^{1} \mathrm{G}^{3}\end{array}$ & & \\
\hline $4 f^{3} \mathrm{D}_{1}$ & 6552.60 & & $5 f^{1} \mathrm{D}_{2}$ & 4439. 72 & \\
\hline
\end{tabular}


TABLE 4.-Observed terms of Si I.-Continued

\begin{tabular}{|c|c|c|c|c|c|}
\hline $\begin{array}{l}\text { Term } \\
\text { symbol }\end{array}$ & $\begin{array}{l}\text { Term } \\
\text { value } \\
\mathrm{cm}^{-1}\end{array}$ & $\Delta \nu$ & $\begin{array}{c}\text { Term } \\
\text { symbol }\end{array}$ & $\begin{array}{l}\text { Term } \\
\text { value } \\
\mathrm{cm}^{-1}\end{array}$ & $\triangle \nu$ \\
\hline $7 s^{3} \mathrm{P}_{0}^{\circ}$ & 4203.00 & & & & \\
\hline $7 s^{3} \mathrm{P}_{\mathrm{i}}$ & 4148. 20 & 54.80 & $6 f^{3} \mathrm{~F}_{2}$ & 3074.50 & \\
\hline $7 s^{3} \mathrm{P}_{2}^{\circ}$ & 3919. 56 & 228. 64 & $8 s^{3} \mathrm{P}_{0}^{\circ}$ & 2989. 95 & 55. 90 \\
\hline $7 s^{1} \mathrm{P}_{1}$ & 3861.50 & 58.06 & $8 s^{3} \mathrm{P}_{1}^{\circ}$ & 2934. 05 & 114. 80 \\
\hline $6 d^{3} \mathrm{D}_{1}^{\circ}$ & 4232. 29 & -86.78 & $\begin{array}{l}8 s^{\circ} \mathrm{P}_{2}^{2} \\
8 s^{1} \mathrm{P}_{1}^{\circ}\end{array}$ & $\begin{array}{l}2819.25 \\
2612.40\end{array}$ & 206. 85 \\
\hline $6 d^{3} \mathrm{D}_{2}^{\circ}$ & 4319.07 & 151. 87 & $7 d^{3} \mathrm{D}_{1}^{\circ}$ & 2869. 10 & \\
\hline $\begin{array}{l}6 d^{3} \mathrm{D}_{3}^{\circ} \\
6 d^{3} \mathrm{P}_{2}^{\circ}\end{array}$ & $\begin{array}{l}4167.20 \\
3897.04\end{array}$ & & $7 d^{3} \mathrm{D}_{2}^{\circ}$ & 2867. 82 & $\begin{array}{r}1.28 \\
61.12\end{array}$ \\
\hline $6 d^{3} \mathrm{P}_{\mathrm{i}}$ & 3806. 14 & -90.90 & $7 d^{3} \mathrm{D}_{3}^{\circ}$ & 2806. 70 & \\
\hline $6 d^{3} \mathrm{P}_{0}^{\circ}$ & 3772.72 & -33.42 & $\begin{array}{l}7 d^{3} \mathrm{~F}_{2}^{\circ} \\
7 d^{3} \mathrm{~F}_{3}^{\circ}\end{array}$ & $\begin{array}{l}2485.39 \\
2389.30\end{array}$ & 96. 09 \\
\hline $6 d^{3} \mathrm{~F}_{2}^{\circ}$ & 3393. 73 & 27.41 & $7 d^{3} \mathrm{~F}_{4}^{\circ}$ & 2162.37 & 226. 93 \\
\hline $\begin{array}{l}6 d^{3} \mathrm{~F}_{3}^{\circ} \\
6 d^{3} \mathrm{~F}_{4}^{\circ}\end{array}$ & $\begin{array}{l}3366.32 \\
3208.54\end{array}$ & 157. 78 & $\begin{array}{l}7 d^{1} \mathrm{D}_{2}^{\circ} \\
7 d^{1} \mathrm{~F}_{3}^{\circ} \\
7 d^{1} \mathrm{P}_{1}^{\circ}\end{array}$ & 2100.45 & \\
\hline $\begin{array}{l}6 d^{1} \mathrm{D}_{2}^{\circ} \\
6 d^{1} \mathrm{P}_{1}^{\circ} \\
6 d^{1} \mathrm{~F}_{3}^{\circ}\end{array}$ & 3587.80 & & $\begin{array}{l}8 d^{3} \mathrm{D}_{3}^{\circ} \\
9 s^{1} \mathrm{P}_{1}^{\circ}\end{array}$ & $\begin{array}{l}1984.65 \\
1858.05\end{array}$ & \\
\hline
\end{tabular}

Comparison of the present list of terms with those reported in the earlier papers will reveal that several corrections and additions have been made. In the $4 p$ group, which are responsible for the intense infrared lines, new values have been assigned to the ${ }^{3} \mathrm{D}$ terms and also to ${ }^{3} \mathrm{P}_{0}$ and ${ }^{1} \mathrm{P}_{1}$. The uncertainties that have attended the identification of some of the $4 f$ and $5 f$ terms, owing to the diffuseness of the lines and the presence of unresolved satellites, have now been removed with the aid of the enclosed arc observations. The numeration of Fowler's odd ${ }^{3} \mathrm{D}$ terms proposed in my earlier paper is here retained despite Robinson's ${ }^{11}$ criticism based on his application of the irregular doublet law to the sequence of isoelectronic spectra beginning with $\mathrm{Si}$ I. The reassignment of Fowler's $3 p^{\prime}{ }^{3} \mathrm{D}^{\circ}$ and ${ }^{3} \mathrm{P}^{\circ}$ terms to the $3 d$ configuration is supported by the discovery of the companion term, $3 d^{3} \mathrm{~F}^{\circ}$, and also of the ${ }^{3} \mathrm{D}^{\circ}$ term at 17200 , which satisfies the combination requirements of a $3 p^{\prime}$ term, but not those of a $3 d$ term. It is to be noted that the odd ${ }^{3} \mathrm{~F}^{\circ}$ terms are all new and make it possible to fill out the complement of terms required for the nd configurations. The extension of the $n d$ terms to higher values of $n$ is due largely to the excellent spectrograms that Shenstone has made of the Schumann region. In particular, these observations have brought to light

\footnotetext{
11 H. A. Robinson, Phys. Rev. 49, 304 (1936).
} 
several new combinations with the $3 p{ }^{1} \mathrm{~S}_{\circ}$ term that are masked by a band structure extending below $2000 \mathrm{~A}$ in observations made with the arc in air.

\section{SERIES AND IONIZATION POTENTIAL OF SiI}

In the light of the present analysis it is now possible to extend to higher members several of Fowler's Si I series. Others must be amended, and still others that are new may be described. Fowler's most reliable series for determination of absolute term values are those from the $n s$ electron. Reference to table 4 will show that the series $3 p^{3} \mathrm{P}-n s^{3} \mathrm{P}^{\circ}$ are now represented by five members. A Ritz formula of the type

$$
\nu=L-R /\left(m+\alpha+\frac{\beta}{m^{2}}\right)^{2},
$$

in which $R_{81}=109735.3$, has been found sufficient to reproduce most of the series of Si I, especially those of the $n s$ and $n f$ configurations, with great fidelity. Applied to the series $3 p^{3} \mathrm{P}_{0}-n s^{3} \mathrm{P}_{1}$ the formula

$$
\nu=65722-R /\left(m-1.8182-\frac{2.0142}{m^{2}}\right)^{2}, m=4,5,6 \ldots
$$

fits the first three members of the series exactly and reproduces the fifth and sixth members with residuals of +25 and $-13 \mathrm{~cm}^{-1}$, respectively. It will be noted that the term value $3 p^{3} \mathrm{P}_{0}=65722$ is in close agreement with Fowler's value, 65765 .

The Ritz type of formula also appears to give a more satisfactory representation of the $n s{ }^{1} \mathrm{P}_{\mathrm{i}}$ terms than does the Hicks formula employed by Fowler. For the series $3 p^{3} \mathrm{P}_{0}-n s^{1} \mathrm{P}_{1}^{0}$ the formula

$$
\nu=65995-R /\left(m-1.7992-\frac{1.6618}{m^{2}}\right)^{2}, m=4,5,6 \ldots
$$

reproduces the observed lines with the residuals $-46,+45,0,0,+15$, respectively. In this case the value of the limit, $L=65995$, represents the distance of $3 p{ }^{3} \mathrm{P}_{0}$ from the $\mathrm{Si}$ in parent term, ${ }^{2} \mathrm{P}_{3 / 2}$, of the $n s^{1} \mathrm{P}_{i}$ terms. According to Fowler's analysis of $\mathrm{Si}^{12}$ the components of the ground state ${ }^{2} \mathrm{P}$ are separated by $287 \mathrm{~cm}^{-1}$. Deduction of this amount from 65995 gives a value of 65708 for the distance separating $3 p{ }^{3} \mathrm{P}_{0}$ from the lowest term of $\mathrm{Si}$ II, in excellent agreement with the result from the $n s^{3} \mathrm{P}_{i}^{\circ}$ terms which converge toward ${ }^{2} \mathrm{P}_{1 / 2}^{\circ}$. Fowler adds also the term $9 s^{1} \mathrm{P}_{1}$ to this series, which is reproduced by the above formula with a residual of $+18 \mathrm{~cm}^{-1}$.

We may expect equally satisfactory results from the nf terms, since they are nearly hydrogenic. Only two members have been found for most of these sequences, but for the $n f^{3} \mathrm{~F}_{2}$ terms there are three members observed. A satisfactory Ritz formula for the series $3 d^{3} \mathrm{~F}_{2}^{\circ}-n f^{3} \mathrm{~F}_{2}$ is

$$
\nu=15915-R /\left(m-0.0569+\frac{0.3256}{m^{2}}\right)^{2}, m=4,5,6 \ldots
$$

\footnotetext{
${ }^{12}$ A. Fowler, Phil. Trans. Roy. Soc. (London) [A] 225, 24 (1925).
} 
The term $3 d^{3} \mathrm{~F}_{2}^{\circ}$ lies 49,851 units above $3 p^{3} \mathrm{P}_{0}$, whence we derive a value 65766 for the ground term of Si I.

Owing to the revisions and additions to the $n d$ terms that have been described above, the sequences in this group are new except for the ${ }^{1} \mathrm{P}^{\circ}$ and ${ }^{1} \mathrm{~F}^{\circ}$ series. They may all be represented with varying degrees of accuracy by properly evaluated Ritz formulas. These are as follows:

Series $3 p{ }^{1} \mathrm{D}_{2}-n d{ }^{1} \mathrm{~F}_{3}^{\circ}$; limit ${ }^{2} \mathrm{P}_{3 / 2}$ in Si II.

$\nu=59583-R_{/}\left(m-0.0326-\frac{0.0511}{m^{2}}\right)^{2}, m=3,4,5 \ldots$

$3 p^{3} \mathrm{P}_{0}=59583-287+6299=65595$.

Series $3 p{ }^{1} \mathrm{D}_{2}-n d{ }^{1} \mathrm{D}_{2}^{\circ}$, limit ${ }^{2} \mathrm{P}_{3 / 2}$ in $\mathrm{Si}$ II $\nu=59710-R /\left(m-0.6384+\frac{0.5728}{m^{2}}\right)^{2}, m=3,4,5 \ldots$ $3 p^{3} \mathrm{P}_{0}=65722$.

Series $3 p{ }^{1} \mathrm{~S}_{0}-n d{ }^{1} \mathrm{P}_{1}$, limit ${ }^{2} \mathrm{P}_{1 / 2}$ in Si II $\nu=50328-R /\left(m-0.0142-\frac{0.0314}{m^{2}}\right)^{2}, m=3,4,5 \ldots$ $3 p^{3} \mathrm{P}_{0}=50328+15394=65722$.

Series $4 p^{3} \mathrm{D}_{3}-n d{ }^{3} \mathrm{~F}_{4}^{\circ}$, limit ${ }^{2} \mathrm{P}_{3 / 2}$ in Si II $\nu=17775-R /\left(m-0.4105+\frac{0.2094}{m^{2}}\right)^{2}, m=3,4,5 \ldots$ $3 p^{3} \mathrm{P}_{0}=17775-287+48264=65752$.

Series $3 p{ }^{3} \mathrm{P}_{1}-n d{ }^{3} \mathrm{~F}_{2}^{\circ}$, limit ${ }^{2} \mathrm{P}_{1 / 2}$ in Si II $\nu=65791-R /\left(m-0.4394+\frac{0.5116}{m^{2}}\right)^{2}, m=3,4,5 \ldots$ $3 p^{3} \mathrm{P}_{0}=65791+77=65868$.

Series $3 p{ }^{3} \mathrm{P}_{2}-n d{ }^{3} \mathrm{D}_{3}^{\circ}$, limit ${ }^{2} \mathrm{P}_{3 / 2}$ in Si II $\nu=65943-R /\left(m-1.2478+\frac{4.8083}{m^{2}}\right)^{2}, m=3,4,5 \ldots$ $3 p^{3} \mathrm{P}_{0}=65943-287+223=65879$.

Series $3 p{ }^{3} \mathrm{P}_{1}-n d{ }^{3} \mathrm{D}_{2}^{\circ}$, limit ${ }^{2} \mathrm{P}_{3 / 2}$ in Si II $\nu=65932-R /\left(m-1.2434+\frac{4.8952}{m^{2}}\right)^{2}, m=3,4,5 \ldots$ $3 p^{3} \mathrm{P}_{0}=65932-287+77=65722$.

Series $3 p{ }^{3} \mathrm{P}_{0}-n d{ }^{3} \mathrm{D}_{1}$, limit ${ }^{2} \mathrm{P}_{3 / 2}$ in Si II $\nu=66107-R /\left(m-1.2480+\frac{4.8158}{m^{2}}\right)^{2}, m=3,4,5 \ldots$. $3 p{ }^{3} \mathrm{P}_{0}=66107-287=65820$.

Series $3 p{ }^{3} \mathrm{P}_{2}-n d{ }^{3} \mathrm{P}_{2}^{\circ}$, Limit ${ }^{2} \mathrm{P}_{1 / 2}$ in Si II $\nu=65500-R /\left(m-0.8165+\frac{4.5540}{m^{2}}\right)^{2}, m=3,4,5 \ldots$ $3 p{ }^{3} \mathrm{P}_{0}=65500+223=65723$.

Series $3 p{ }^{3} \mathrm{P}_{0}-n d{ }^{3} \mathrm{P}_{1}$, limit ${ }^{2} \mathrm{P}_{1 / 2}$ in Si II $\nu=66001-R /\left(m-0.9928+\frac{6.8477}{m^{2}}\right)^{2}, m=3,4,5 \ldots$ $3 p{ }^{3} \mathrm{P}_{0}=66001-287=65714$. 
An inspection of the results presented in the foregoing paragraphs will show that the component series of the $n s$ terms approach their limits according to the theory of Hund. ${ }^{13}$ With the $n d$ series, however, another procedure is followed. This was already forecast by Fowler, who noted that the $n d{ }^{1} \mathrm{P}_{1}^{\circ}$ terms of $\mathrm{Si}$ I converge toward ${ }^{2} \mathrm{P}_{1 / 2}^{\circ}$ instead of ${ }^{2} \mathrm{P}_{3 / 2}^{\circ}$ of $\mathrm{Si}$ II. This behavior is confirmed by the present analysis and, in addition, it is seen that the $n d^{3} \mathrm{P}_{2}^{\circ}$ terms also converge toward ${ }^{2} \mathrm{P}_{1 / 2}^{\circ}$ of $\mathrm{Si}$ II, while the three components of the $n d$ ${ }^{3} \mathrm{D}^{\circ}$ group converge toward ${ }^{2} \mathrm{P}_{3 / 2}^{\circ}$. This is shown graphically in figure 2 . Although the coordination of individual series with their proper limits presented herein differs with Hund's theoretical scheme, nevertheless, the quantum requirements are fulfilled that series terms with inner quantum numbers, 4, 3, 3, 2, 2, 1, 1, 0 converge toward ${ }^{2} \mathrm{P}_{3 / 2}^{\circ}$ and those with inner quantum numbers $3,2,2,1$, converge toward ${ }^{2} \mathrm{P}_{1 / 2}^{\circ}$. The sequences of $n p$ and $n f$ terms have for the most part only two mem-

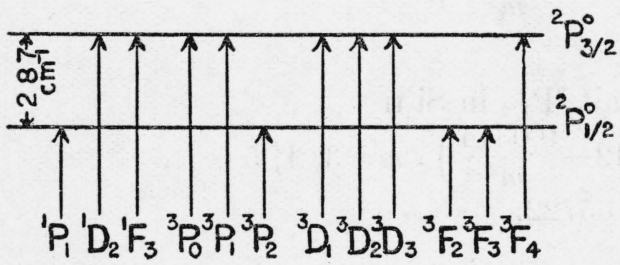

Figure 2.-Coordination of component series of the d-electron with limits in $\mathrm{Si}$ II.

bers and are therefore not adequate for the coordination of their components with limits in Si II.

From the various series described above a mean value of $65743 \mathrm{~cm}^{-1}$ is found for the separation of $3 p^{3} \mathrm{P}^{\circ}$ of $\mathrm{Si} \mathrm{I}$ and $3 p{ }^{2} \mathrm{P}_{01 / 2}^{\circ}$ of $\mathrm{Si}$ II, corresponding, therefore, to an ionization potential of 8.11 volts. This value differs only by $22 \mathrm{~cm}^{-1}$ from Fowler's value of 65765 .

\section{SILICON IN THE SUN}

The presence of silicon in the sun has long been established through the $4 s-5 p$ lines in the yellow and the $3 p-4 s$ lines in the violet. Subsequently, identification of red and infrared solar lines with silicon was made by Meggers ${ }^{14}$ with the aid of unpublished National Bureau of Standards observations. As more extensions of both solar and silicon spectra became available for comparison, it was found that nearly all the stronger lines emitted by the $\mathrm{Si}$ arc have their counterparts in the sun. Such a comparison is set forth in table 1, in the third column of which are given the values of the solar wave lengths taken either from the Revision of Rowland's Preliminary Table ${ }^{15}$ or from Babcock's published ${ }^{16}$ and unpublished lists of infrared solar lines.

However, the silicon atom is capable of emitting more lines than those listed in tables 1 and 2. Very few of the multiplets that have been described in this paper are emitted with their full complement of lines by the arc sources described above. In table 5 are listed unobserved lines calculated from term combinations and with them the nearest solar lines. A group of lines in the blue-green may be expected from the $4 s-6 p$ transition. The $6 p$ terms have not yet been detected in the laboratory but the lines resulting from their combination with the $4 s$ terms are in all probability present in the sun.

\footnotetext{
13 F. Hund, Linienspektren, p. 184 (Julius Springer, Berlin, 1927).

14 W. F. Meggers, Pub. Allegheny Obs., 6, 13 (1919).

15 Carnegie Institution of Washington Publication 396, (1928).

${ }^{10} \mathrm{H}$. D. Babcock, Trans. Int. Astron. Union 5, 90 (1935) Astrophys. J. 83, 103 (1936).
} 
TABLE 5.-Comparison of computed Si I lines with solar lines

\begin{tabular}{|c|c|c|c|c|}
\hline $\begin{array}{l}\text { Computed } \\
\text { wave length } \\
\text { of } \mathrm{Si} \mathrm{I}\end{array}$ & $\begin{array}{l}\text { Wave } \\
\text { length } \\
\text { in sun }\end{array}$ & Solar intensity & $\begin{array}{l}\text { Wave num- } \\
\text { ber } \nu\end{array}$ & Term combination \\
\hline $\begin{array}{l}11890.29 \\
10976.25 \\
10616.59 \\
10580.20 \\
10179.38\end{array}$ & $\begin{array}{l}\text { 0. } 51 \\
\text { 6. } 30 \\
\text { 6. } 71 \\
\text { 0. } 61 \\
\text { 9. } 36\end{array}$ & $\begin{array}{r}-1 \\
-1 \\
1 \\
-3 \\
-1\end{array}$ & $\begin{array}{l}8407.92 \\
9108.09 \\
9416.64 \\
9449.04 \\
9821.10\end{array}$ & $\begin{array}{l}4 s^{1} \mathrm{P}_{1}-4 p^{3} \mathrm{~S}_{1} \\
4 p^{3} \mathrm{D}_{3}-4 d^{3} \mathrm{~F}_{2}^{\circ} \\
4 p^{1} \mathrm{P}_{1}-4 d^{3} \mathrm{P}_{1} \\
4 p^{1} \mathrm{P}_{1}-4 d^{3} \mathrm{P}_{0}^{0} \\
4 p^{3} \mathrm{~S}_{1}-6 s^{3} \mathrm{P}_{0}^{0}\end{array}$ \\
\hline $\begin{array}{r}10125.22 \\
10025.45 \\
10001.15 \\
9453.00 \\
9405.71\end{array}$ & $\begin{array}{l}\text { 4. } 92 \\
\text { 5. } 86 \\
\text { 1. } 31 \\
\text { 3. } 12 \\
\text { 5. } 80\end{array}$ & $\begin{array}{l}-2 \\
2 n \\
-1 \\
-2 \\
0 N\end{array}$ & $\begin{array}{r}9873.62 \\
9971.87 \\
9996.15 \\
10575.79 \\
10628.93\end{array}$ & $\begin{array}{l}4 p^{3} \mathrm{~S}_{1}-6 s^{3} \mathrm{P}_{1} \\
4 p^{3} \mathrm{P}_{1}-5 d^{3} \mathrm{D}_{2} \\
4 p^{3} \mathrm{P}_{1}-5 d^{3} \mathrm{D}_{1}^{\circ} \\
4 p^{3} \mathrm{P}_{1}-6 s^{1} \mathrm{P}_{1} \\
4 p^{3} \mathrm{D}_{3}-4 d^{1} \mathrm{~F}_{3}^{\circ}\end{array}$ \\
\hline $\begin{array}{l}9393.43 \\
9078.16 \\
8928.37 \\
8780.00 \\
8766.24\end{array}$ & $\begin{array}{l}\text { 3. } 44 \\
8.28 \\
8.17 \\
0.17 \\
\text { 6. } 42\end{array}$ & $\begin{array}{l}-3 \\
\quad 1 d ? \mathrm{C} \\
-3 \\
-3 \\
-1\end{array}$ & $\begin{array}{l}10642.82 \\
11012.42 \\
11197.18 \\
11386.40 \\
11404.27\end{array}$ & $\begin{array}{c}4 p^{3} \mathrm{~S}_{1}-5 d^{3} \mathrm{P}_{0}^{\circ} \\
4 p^{3} \mathrm{D}_{1}-5 d^{3} \mathrm{D}_{2}^{\circ} \\
4 p{ }^{1} \mathrm{~S}_{0}-8 s^{3} \mathrm{P}_{1}^{\circ} \\
4 p p^{1} \mathrm{D}_{2}-6 d^{3} \mathrm{D}_{3}^{\circ} \\
4 p^{1} \mathrm{D}_{2}-5 d^{1} \mathrm{P}_{1}^{\circ}\end{array}$ \\
\hline $\begin{array}{l}\text { 8703. } 73 \\
8680.22 \\
8679.05 \\
8667.07 \\
8579.13\end{array}$ & $\begin{array}{l}\text { 3. } 73 \\
0.10 \\
8.95 \\
7.37 \\
9.08\end{array}$ & $\begin{array}{l}-2 N, \mathrm{Mn} \\
-1 \\
-2 \mathrm{~S} \\
-1 \\
-2\end{array}$ & $\begin{array}{l}11486.17 \\
11517.29 \\
11518.83 \\
11533.96 \\
11653.00\end{array}$ & $\begin{array}{r}4 p^{3} \mathrm{D}_{1}-6 s^{3} \mathrm{P}_{2} \\
4 p{ }^{3} \mathrm{P}_{2}-5 d^{3} \mathrm{~F}_{3}^{\circ} \\
4 p{ }^{1} \mathrm{~S}_{0}-8 s^{1} \mathrm{P}_{1}^{\circ} \\
4 p^{3} \mathrm{D}_{2}-6 s^{1} \mathrm{P}_{1}^{\circ} \\
4 p^{3} \mathrm{D}_{3}-5 d^{3} \mathrm{P}_{2}^{\circ}\end{array}$ \\
\hline $\begin{array}{l}8576.53 \\
8550.46 \\
8511.40 \\
8510.16 \\
8492.00\end{array}$ & $\begin{array}{l}\text { 6. } 48 \\
0.37 \\
\text { 1. } 45 \\
\text { 0. } 25 \\
\text { 2. } 08\end{array}$ & $\begin{array}{r}-3 \\
1 \\
-3 \\
-1 \\
-2\end{array}$ & $\begin{array}{l}11656.53 \\
11692.07 \\
11745.75 \\
11747.43 \\
11772.50\end{array}$ & $\begin{array}{l}4 p^{1} \mathrm{D}_{2}-6 d^{3} \mathrm{P}_{2}^{\circ} \\
4 p^{1} \mathrm{D}_{2}-7 s^{1} \mathrm{P}_{1}^{0} \\
4 p^{3} \mathrm{D}_{2}-5 d^{3} \mathrm{P}_{1}^{\circ} \\
4 p^{1} \mathrm{D}_{2}-6 d^{3} \mathrm{P}_{1}^{\circ} \\
4 p^{1} \mathrm{P}_{1}-5 d^{3} \mathrm{D}_{1}^{\circ}\end{array}$ \\
\hline $\begin{array}{l}8461.51 \\
8337.93 \\
8305.75 \\
8198.48 \\
8195.46\end{array}$ & $\begin{array}{l}\text { 1. } 47 \\
\text { 7. } 92 \\
\text { 5. } 62 \\
\text { 8. } 28 \\
\text { 5. } 45\end{array}$ & $\begin{array}{l}-3 \\
-3 \\
-1 \\
-3 \\
-2\end{array}$ & $\begin{array}{l}11814.97 \\
11990.10 \\
12036.55 \\
12194.04 \\
12198.52\end{array}$ & $\begin{array}{c}4 p^{3} \mathrm{D}_{2}-5 d^{3} \mathrm{P}_{2}^{\circ} \\
4 p^{3} \mathrm{D}_{1}-5 d^{3} \mathrm{P}_{1}^{\circ} \\
4 p^{3} \mathrm{D}_{3}-5 d^{1} \mathrm{D}_{2}^{\circ} \\
4 p{ }^{3} \mathrm{~S}_{1}-5 d^{1} \mathrm{P}_{1}^{\circ} \\
4 p^{3} \mathrm{D}_{2}-5 d^{1} \mathrm{D}_{2}^{\circ}\end{array}$ \\
\hline $\begin{array}{l}8145.61 \\
8086.12 \\
8074.58 \\
8070.64 \\
7976.65\end{array}$ & $\begin{array}{l}\text { 5. } 48 \\
\text { 6. } 18 \\
\text { 4. } 43 \\
\text { 0. } 62 \\
\text { 6. } 59\end{array}$ & $\begin{array}{l}-1 \mathrm{Fe} \\
-3 \\
-2 \mathrm{Atm} ? \\
-1 \\
-1 \mathrm{Atm} ?\end{array}$ & $\begin{array}{l}\text { 12273. } 18 \\
\text { 12363. } 38 \\
\text { 12381. } 14 \\
\text { 12387. } 19 \\
\text { 12533. } 15\end{array}$ & $\begin{array}{c}4 p{ }^{1} \mathrm{~S}_{0}-7 d^{1} \mathrm{P}_{1}^{\circ} \\
4 p^{3} \mathrm{P}_{1}-6 d^{3} \mathrm{D}_{2}^{\circ} \\
4 p^{3} \mathrm{D}_{3}-5 d^{3} \mathrm{~F}_{2}^{\circ} \\
4 p{ }^{3} \mathrm{P}_{2}-6 d^{3} \mathrm{D}_{3}^{\circ} \\
4 p^{3} \mathrm{P}_{1}-5 d^{1} \mathrm{P}_{1}^{\circ}\end{array}$ \\
\hline $\begin{array}{l}\text { 7898. } 37 \\
7855.83 \\
7725.16 \\
7523.11 \\
7518.50\end{array}$ & $\begin{array}{l}\text { 8. } 38 \\
\text { 5. } 82 \\
\text { 5. } 17 \\
\text { 3. } 22 \\
\text { 8. } 66\end{array}$ & $\begin{array}{l}-3 \\
-3 \text { Co } \\
-1 \\
-3 \\
-3 N\end{array}$ & $\begin{array}{l}\text { 12657. } 35 \\
12725.90 \\
12941.17 \\
13288.72 \\
13296.87\end{array}$ & $\begin{array}{r}4 p^{3} \mathrm{P}_{2}-6 d^{3} \mathrm{P}_{2}^{\circ} \\
4 p{ }^{1} \mathrm{P}_{1}-5 d^{3} \mathrm{P}_{1}^{\circ} \\
4 p^{1} \mathrm{D}_{2}-8 s^{1} \mathrm{P}_{1}^{\circ} \\
4 p{ }^{3} \mathrm{P}_{1}-6 d^{3} \mathrm{~F}_{2}^{\circ} \\
4 p^{3} \mathrm{D}_{3}-5 d^{1} \mathrm{~F}_{3}^{\circ}\end{array}$ \\
\hline $\begin{array}{l}\text { 7504. } 60 \\
7486.67 \\
7482.25 \\
7458.44 \\
7456.00\end{array}$ & $\begin{array}{l}\text { 4. } 61 \\
\text { 6. } 67 \\
\text { 2. } 21 \\
\text { 8. } 38 \\
\text { 6. } 35\end{array}$ & $\begin{array}{l}-3 \\
-2 \\
-1 \\
-2 \\
-3\end{array}$ & $\begin{array}{l}13321.55 \\
13353.39 \\
13361.29 \\
13403.93 \\
13408.33\end{array}$ & $\begin{array}{c}4 p^{3} \mathrm{D}_{2}-6 d^{3} \mathrm{D}_{2}^{2} \\
4 p{ }^{3} \mathrm{~S}_{1}-8 s^{3} \mathrm{P}_{0}^{\circ} \\
4 p{ }^{1} \mathrm{P}_{1}-5 d^{3} \mathrm{~F}_{2}^{\circ} \\
4 p p^{3} \mathrm{D}_{1}-6 d^{3} \mathrm{D}_{2}^{2} \\
4 p^{3} \mathrm{D}_{2}-6 d^{3} \mathrm{D}_{1}^{\circ}\end{array}$ \\
\hline
\end{tabular}


TABLE 5.-Comparison of computed $\mathrm{Si}$ I lines with solar lines-Continued

\begin{tabular}{|c|c|c|c|c|}
\hline $\begin{array}{l}\text { Computed } \\
\text { wave length } \\
\text { of Si I }\end{array}$ & $\begin{array}{l}\text { Wave } \\
\text { length } \\
\text { in sun }\end{array}$ & Solar intensity & $\begin{array}{l}\text { Wave num- } \\
\text { ber } \nu\end{array}$ & Term combination \\
\hline $\begin{array}{l}\text { 7455. } 44 \\
7431.17 \\
7410.14 \\
7409.53 \\
7392.18\end{array}$ & $\begin{array}{l}\text { 5. } 39 \\
1.14 \\
9.99 \\
9.69 \\
2.13\end{array}$ & $\begin{array}{l}-3 \\
-3 \\
-3 \\
-3 \\
-3 N\end{array}$ & $\begin{array}{l}\text { 13409. } 29 \\
\text { 13453. } 12 \\
\text { 13491. } 32 \\
\text { 13492. } 42 \\
13524.09\end{array}$ & $\begin{array}{c}4 p^{3} \mathrm{~S}_{1}-8 s^{3} \mathrm{P}_{1}^{\circ} \\
4 p^{1} \mathrm{D}_{2}-7 d^{1} \mathrm{~F}_{3}^{\circ} \\
4 p^{3} \mathrm{D}_{2}-5 d^{1} \mathrm{P}_{1}^{\circ} \\
4 p^{3} \mathrm{D}_{2}-7 s^{3} \mathrm{P}_{1}^{\circ} \\
4 p^{3} \mathrm{~S}_{1}-8 s^{3} \mathrm{P}_{2}^{\circ}\end{array}$ \\
\hline $\begin{array}{l}7367.75 \\
7365.16 \\
7364.56 \\
7360.87 \\
7278.60\end{array}$ & $\begin{array}{l}\text { 7. } 76 \\
\text { 5. } 30 \\
4.65 \\
\text { 0. } 81 \\
\text { 8. } 52\end{array}$ & $\begin{array}{l}-3 \\
0 \text { Atm } \\
-3 N \\
-3 \\
-2\end{array}$ & $\begin{array}{l}\text { 13568. } 92 \\
13573.70 \\
13574.80 \\
13581.61 \\
13735.14\end{array}$ & $\begin{array}{l}4 p^{1} \mathrm{D}_{2}-7 d^{1} \mathrm{~F}_{3}^{\circ} \\
4 p^{3} \mathrm{D}_{1}-5 d^{1} \mathrm{P}_{1}^{\circ} \\
4 p^{3} \mathrm{D}_{1}-7 s^{3} \mathrm{P}_{1}^{\circ} \\
4 p^{3} \mathrm{D}_{3}-6 d^{3} \mathrm{P}_{2}^{\circ} \\
4 p^{3} \mathrm{P}_{2}-8 s^{3} \mathrm{P}_{2}^{\circ}\end{array}$ \\
\hline $\begin{array}{l}\text { 7114. } 05 \\
7097.80 \\
7072.59 \\
7017.19 \\
6865.41\end{array}$ & $\begin{array}{l}\text { 4. } 19 \\
\text { 7. } 68 \\
\text { 2. } 81 \\
\text { 7. } 33 \\
\text { 5. } 46\end{array}$ & $\begin{array}{l}-3 \mathrm{Atm} ? \\
-3 \\
-3 \\
-1 \mathrm{Nd} \\
-3\end{array}$ & $\begin{array}{l}14052.82 \\
14084.92 \\
14135.20 \\
14246.89 \\
14561.76\end{array}$ & $\begin{array}{l}4 p^{3} \mathrm{D}_{2}-6 d^{1} \mathrm{D}_{2}^{\circ} \\
4 p^{3} \mathrm{D}_{3}-6 d^{3} \mathrm{~F}_{2}^{\circ} \\
4 p^{3} \mathrm{D}_{1}-6 d^{1} \mathrm{D}_{2}^{\circ} \\
4 p^{3} \mathrm{D}_{2}-6 d^{3} \mathrm{~F}_{2}^{\circ} \\
4 p^{1} \mathrm{P}_{1}-6 d^{3} \mathrm{P}_{2}^{\circ}\end{array}$ \\
\hline $\begin{array}{l}6842.35 \\
6819.68 \\
6813.85 \\
6807.29 \\
6767.92\end{array}$ & $\begin{array}{l}\text { 2. } 38 \\
\text { 9. } 86 \\
\text { 3. } 92 \\
\text { 7. } 39 \\
\text { 8. } 01\end{array}$ & $\begin{array}{l}-3 d ? \\
-2 d ? \\
-3 N \\
-3 d ? \\
-3\end{array}$ & $\begin{array}{l}14610.83 \\
14659.40 \\
14671.95 \\
14686.08 \\
14771.52\end{array}$ & $\begin{array}{l}4 p^{3} \mathrm{D}_{3}-7 d^{3} \mathrm{D}_{2}^{\circ} \\
4 p^{3} \mathrm{D}_{3}-8 s^{3} \mathrm{P}_{2}^{\circ} \\
4 p^{3} \mathrm{D}_{3}-7 d^{3} \mathrm{D}_{3}^{\circ} \\
4 p^{1} \mathrm{P}_{1}-6 d^{3} \mathrm{P}_{0}^{\circ} \\
4 p^{3} \mathrm{D}_{2}-7 d^{3} \mathrm{D}_{1}^{\circ}\end{array}$ \\
\hline $\begin{array}{l}6767.33 \\
6739.45 \\
6730.39 \\
6729.80 \\
6722.65\end{array}$ & $\begin{array}{l}\text { 7. } 36 \\
9.53 \\
0.32 \\
9.75 \\
\text { 2. } 72\end{array}$ & $\begin{array}{l}-3 d ? \\
0 \mathrm{Fe} \\
-2 N \\
-2 N, \mathrm{Cr} ? \\
-3 N\end{array}$ & $\begin{array}{l}\text { 14772. } 80 \\
14833.92 \\
14853.90 \\
14855.18 \\
14871.00\end{array}$ & $\begin{array}{l}4 p^{3} \mathrm{D}_{2}-7 d^{3} \mathrm{D}_{2}^{\circ} \\
4 p^{3} \mathrm{D}_{2}-7 d^{3} \mathrm{D}_{3}^{\circ} \\
4 p^{3} \mathrm{D}_{1}-7 d^{3} \mathrm{D}_{1}^{\circ} \\
4 p^{3} \mathrm{D}_{1}-7 d^{3} \mathrm{D}_{2}^{\circ} \\
4 p^{1} \mathrm{P}_{1}-6 d^{1} \mathrm{D}_{2}^{\circ}\end{array}$ \\
\hline $\begin{array}{l}6500.92 \\
6462.82 \\
6439.55 \\
6308.84 \\
6022.16\end{array}$ & $\begin{array}{l}0.85 \\
2.98 \\
9.58 \\
\text { 8. } 84 \\
2.23\end{array}$ & $\begin{array}{l}-3 \\
-3 \\
-3 \\
-2 N \\
-3\end{array}$ & $\begin{array}{l}15378.20 \\
15468.85 \\
15524.75 \\
15846.40 \\
16600.75\end{array}$ & $\begin{array}{l}4 p^{3} \mathrm{D}_{3}-7 d^{1} \mathrm{~F}_{3}^{\circ} \\
4 p^{1} \mathrm{P}_{1}-8 s^{3} \mathrm{P}_{0}^{0} \\
4 p^{1} \mathrm{P}_{1}-8 s^{3} \mathrm{P}_{1}^{\circ} \\
4 p^{1} \mathrm{P}_{1}-8 s^{1} \mathrm{P}_{1}^{\circ} \\
4 p^{1} \mathrm{P}_{1}-9 s^{1} \mathrm{P}_{1}^{\circ}\end{array}$ \\
\hline 3345.54 & 5. 49 & -2 & 29881. 96 & $3 p^{1} \mathrm{~S}_{0}-3 d^{3} \mathrm{D}_{1}$ \\
\hline
\end{tabular}




\section{LINES OF Si II}

Several lines of Si II were measured repeatedly while the work described in this paper was in progress. The mean values of these determinations are given at this time in table 6 to complete the observational record.

TABLE 6.-Wave lengths of some Si II lines

\begin{tabular}{|c|c|c|c|c|}
\hline $\begin{array}{l}\text { Wave } \\
\text { length }\end{array}$ & Intensity & $\begin{array}{c}\text { Wave } \\
\text { number }\end{array}$ & $\Delta \nu$ & Term combination \\
\hline $\begin{array}{l}6371.33 \\
6347.07\end{array}$ & $\begin{array}{l}15 \\
30\end{array}$ & $\begin{array}{l}15690.98 \\
15750.96\end{array}$ & 59. 98 & $\begin{array}{l}4 s^{2} \mathrm{~S}_{01 / 2}-4 p^{2} \mathrm{P}_{01 / 2}^{\circ} \\
4 s^{2} \mathrm{~S}_{01 / 2}-4 p^{2} \mathrm{P}_{11 / 2}^{\circ}\end{array}$ \\
\hline $\begin{array}{l}\text { 1817. } 42 \\
1816.94 \\
1808.01\end{array}$ & $\begin{array}{l}2 \\
8 \\
7\end{array}$ & $\begin{array}{l}55023.05 \\
55037.58 \\
55309.43\end{array}$ & 286. 38 & $\begin{array}{l}3 p^{2} \mathrm{P}_{11 / 2}^{o}-3 p^{\prime}{ }^{2} \mathrm{D}_{11 / 2} \\
3 p^{2} \mathrm{P}_{11 / 2}^{o}-3 p^{\prime}{ }^{2} \mathrm{D}_{21 / 2} \\
3 p^{2} \mathrm{P}_{01 / 2}^{o}-3 p^{\prime}{ }^{2} \mathrm{D}_{1 / 2}\end{array}$ \\
\hline $\begin{array}{l}1533.44 \\
1526.70\end{array}$ & $\begin{array}{l}5 \\
4\end{array}$ & $\begin{array}{l}65212.85 \\
65500.75\end{array}$ & 287. 90 & $\begin{array}{l}3 p^{2} \mathrm{P}_{11 / 2}^{\circ}-4 s^{2} \mathrm{~S}_{01 / 2} \\
3 p^{2} \mathrm{P}_{01 / 2}^{\circ}-4 s^{2} \mathrm{~S}_{01 / 2}\end{array}$ \\
\hline
\end{tabular}

The work described in the foregoing pages would not have been possible without the friendly cooperation of various individuals. As stated above, R. L. Templin, of the Aluminum Research Laboratories, and J. H. Critchett, of the Union Carbide Research Laboratories, have both contributed generous samples of highly purified silicon for use as electrode material. A. G. Shenstone, Palmer Physical Laboratory, Princeton University, has made the necessary observations for a study of the ultraviolet spectrum. Charlotte Moore-Sitterly, Princeton University, and H. D. Babcock, Mount Wilson Observatory, have made available to me their unpublished lists of infrared solar wave lengths; and finally, W. H. Swanger, A. J. Dornblatt, and E. F. Webb, of this Bureau, have been instrumental in casting and shaping the electrodes used in the arc lamps. It is a pleasure for me to express to each of them my appreciation for his contribution to this work.

Washington, May 10, 1938. 